\title{
CONJUGACY CLASSES WHOSE SQUARE IS AN INFINITE SYMMETRIC GROUP
}

\author{
GADI MORAN
}

\begin{abstract}
Let $X_{\nu}$ be the set of all permutations $\xi$ of an infinite set $A$ of cardinality $\aleph_{\nu}$ with the property: every permutation of $A$ is a product of two conjugates of $\xi$. The set $X_{0}$ is shown to be the set of permutations $\xi$ satisfying one of the following three conditions:

(1) $\xi$ has at least two infinite orbits.

(2) $\xi$ has at least one infinite orbit and infinitely many orbits of a fixed finite size $n$.

(3) $\xi$ has: no infinite orbit; infinitely many finite orbits of size $k, l$ and $k+l$ for some positive integers $k, l$; and infinitely many orbits of size $>2$. It follows that $\xi \in X_{0}$ iff some transposition is a product of two conjugates of $\xi$, and $\xi$ is not a product $\sigma i$, where $\sigma$ has a finite support and $i$ is an involution.

For $\nu>0, \xi \in X_{\nu}$ iff $\xi$ moves $\aleph_{\nu}$ elements, and satisfies (1), (2) or $\left(3^{\prime}\right)$, where $\left(3^{\prime}\right)$ is obtained from (3) by omitting the requirement that $\xi$ has infinitely many orbits of size $>2$. It follows that for $\nu>0, \xi \in X_{\nu}$ iff $\xi$ moves $\aleph_{\nu}$ elements and some transposition is the product of two conjugates of $\xi$.

The covering number of a subset $X$ of a group $G$ is the smallest power of $X$ (if any) that equals $G[\mathrm{AH}]$. These results complete the classification of conjugacy classes in infinite symmetric groups with respect to their covering number.
\end{abstract}

\section{BACKGROUND AND RESULTS}

Let $A$ be a countably infinite set, and let $S=S_{A}$ denote the group of all permutations of $A$. For $X, Y \subseteq S$ let $X \cdot Y=\{\xi \eta: \xi \in X, \eta \in Y\}$ and let $X^{1}=X, X^{n+1}=X^{n} \cdot X$. For $\xi \in S$ let $[\xi]=\left\{\eta \xi \eta^{-1}: \eta \in S\right\}$ denote the conjugacy class (coc) of $\xi$ in $S$, and consider the equation

$$
[\xi]^{2}=S .
$$

Let $X_{0} \subseteq S$ be the set of all $\xi \in S$ satisfying (1). We shall establish

Theorem 1. Let $\xi \in S$. Then $\xi \in X_{0}$ if and only if $\xi$ satisfies one of the following three mutually exclusive conditions:

(1) $\xi$ has at least two infinite orbits.

Received by the editors August 3, 1987.

1980 Mathematics Subject Classification (1985 Revision). Primary 20B30, 20B07; Secondary 20E34, 05 C25. 
(2) (a) $\xi$ has precisely one infinite orbit.

(b) $\xi$ has infinitely many orbits of a fixed finite size $n$.

(3) (a) $\xi$ has no infinite orbit.

(b) For some positive integers $k, l, \xi$ has infinitely many orbits of size $n$, for $n=k, n=l$ and $n=k+l$.

(c) $\xi$ has infinitely many orbits of size greater than 2 .

Much of the knowledge and technique accumulated in evaluating products of cocs in $S$ is used in a full proof of Theorem 1. Recently Droste [D3] combined these to establish Theorem 1 for those $\xi \in S$ which have at least one infinite orbit, or fix infinitely many elements. To complete the argument one more new theorem is needed, namely, Theorem A, which we state below.

Let INF $\subseteq S$ be the set of all $\xi \in S$ that move infinitely many elements. Call $\xi \in \mathrm{INF}$ almost involution if $\xi$ has no infinite orbit and finitely many finite orbits of length $>2$ (hence $\xi$ has infinitely many orbits of size 2 ). Let $\mathrm{AI} \subseteq \mathrm{INF}$ be the set of all almost involutions.

Theorem A. Let $\xi, \eta \in \mathrm{INF}$ have no infinite orbit, but infinitely many finite orbits of length at least $k, k \in\{3,4\}$.

(1) If $k=3$ then INF-AI $\subseteq[\xi] \cdot[\eta]$.

(2) If $k=4$ then INF $\subseteq[\xi] \cdot[\eta]$.

(3) The value $k=3(k=4)$ in (1) (in (2)) cannot be reduced.

Our tool for establishing Theorem A is the use of bicolored planar maps, first introduced in [M4] (see also [M5]). This technique is reviewed in $\S 2$.

A brief history about the search for $X_{0}$ is in order. In 1951 Ore [O] proved that every member of $S$ is a commutator. Since $[\xi]=\left[\xi^{-1}\right]$ holds in $S$, $\eta=\zeta \xi \zeta^{-1} \xi^{-1}$ implies $\eta \in[\xi]^{2}$ and so Ore's result shows that $S$ is a union of squares of conjugacy classes.

In 1960 Gray [G] showed that if $\xi \in S$ has infinitely many infinite orbits and no finite orbits, then $S=[\xi]^{2}$, and so $X_{0} \neq \varnothing$. He also noted that the obvious necessary condition $\xi \in \mathrm{INF}$ is not sufficient for $\xi \in X_{0}$.

In 1973 Bertram [B] showed that if $\xi \in S$ has infinitely many orbits of length $n$ for $n=1,2,3$ but no other orbit, then $\xi \in X_{0}$. He used it to show that $[\xi]^{4}=S$ for every $\xi \in \mathrm{INF}$ (see also [DG1]), and conjectured that actually $[\xi]^{3}=S$ for $\xi \in$ INF.

Shortly afterwards it was noted [M1; M2, (2), p. 76] (see also [DG1]) that if $\rho_{0} \in S$ is a fixed-point-free involution, $R_{0}=\left[\rho_{0}\right]$, then $R_{0}^{3} \neq S$ (see [M1, M6] for the actual evaluation of $R_{0}^{3}$ ). However, twelve years later Droste [D2] showed that $R_{0}$ is the only coc in $S$ for which Bertram's conjecture fails.

In 1980 we determined [M3] the set of $\xi \in S$ with parity features, i.e. with the property that $[\xi]^{2}$ contains no finitary odd permutations $(\xi \in S$ is called finitary if it moves only finitely many elements). We now formulate as Theorem 
2 an unpublished observation made in the course of that investigation. This result turns out to have much significance in the present context.

Let $Y_{0} \subseteq S$ be the set of $\eta$ 's satisfying:

$[\eta]^{2}$ contains a transposition.

Theorem 2. Let $\xi \in S$. Then $\xi \in Y_{0}$ if and only if $\xi$ satisfies one of the three mutually exclusive conditions (1), (2), or (3)(a) and (3)(b) mentioned in Theorem 1.

A proof of Theorem 2 is given in $\S 1$.

Comparing Theorems 1 and 2 we conclude:

Corollary 3. Let $\xi \in \mathrm{INF}$. Then $[\xi]^{2}=S$ if and only if:

(i) $[\xi]^{2}$ contains a transposition.

(ii) $\xi$ is not almost involution.

The first indication that $X_{0}$ and $Y_{0}$ are closely related was given in 1982 in Droste's paper [D1]. Indeed, Theorem 1 of [D1], implies that if $\xi \in S$ has at least one infinite orbit, then $\xi \in X_{0}$ if and only if $\xi \in Y_{0}$. Other interesting results of this paper fit into the research on covering numbers of groups that was carried out intensively at the same time (see [AH]). If $C$ is a coc in a group $G$, we write $\operatorname{cn}(C)=n$ if $n$ is the smallest positive integer such that $C^{n}=G \cdot \operatorname{cn}(C)$ is called the covering number of $G$ by $C$ and, when $G$ is fixed in context, the covering number of $C$. If no such integer exists, we write $\operatorname{cn}(C)=\omega$. During 1980-1983 these numbers were computed for various cocs in various groups, and the effort to obtain them in simple factors of the decomposition chain of infinite symmetric groups led us to the discovery and use of the method of bicolored planar maps [ACM, M4, M5]. Droste's work [D1] has immediate relevance to this work as well (see [D1, §5]).

Combining his old results on products of cocs in $S$ with the fresh ones obtained in the course of research on the covering numbers, Droste [D2] obtained the remarkable result mentioned above, that $R_{0}$ is the only $\operatorname{coc} C$ in $S$ with $\operatorname{cn}(C)=4$. Theorem 1 determines the $\operatorname{cocs} C$ in $S$ with $\operatorname{cn}(C)=2$. The remaining cocs in INF-including those with Parity Features (see [M3])-all have covering number 3 . Since $\operatorname{cn}(C)=\omega$ for every finitary $\operatorname{coc} C$, we know now $\operatorname{cn}(C)$ for every $\operatorname{coc} C$ in $S$.

Applying this same body of knowledge and some more recent acquaintance with products of cocs in finite symmetric groups due independently to Boccara [Bo] and Dvir [Dv], Droste then found that $X_{0}$ and $Y_{0}$ are indeed very close. In [D3, Theorem 2], he shows that if $\xi$ has no infinite orbit, and infinitely many fixed points, then $\xi \in X_{0}$ iff $\xi \in Y_{0}$ and $\xi$ is not an almost-involution. The complete determination of $X_{0}$ is reduced, then, to familiarity with those $\xi$ 's in $X_{0}$ that have no infinite orbit, and only finitely many fixed points. Now, $X_{0} \subseteq Y_{0}$ and Theorem 2 further reduce the search to the ones that satisfy 
conditions (3)(a) and (3)(b), stated in Theorem 1. Theorem A provides us with the missing link, and closes the argument for Theorem 1.

Let us review some relevant results. For an arbitrary group $G$, let $X_{G}$ denote the set of $g \in G$ satisfying $[g]^{2}=G$. For an arbitrary ordinal $\nu$, let $S_{\nu}$ denote the group of all permutations of a set of cardinality $\aleph_{\nu}$, and let $X_{\nu}=X_{S_{\nu}}$. Let $S_{\nu}^{\tau} \subseteq S_{\nu}$ be the normal subgroup of $S_{\nu}$ consisting of permutations moving less than $\aleph_{\tau}$ elements and let $H_{\tau}^{\nu}=S_{\nu}^{\tau+1} / S_{\nu}^{\tau}, \tau=0, \ldots, \nu$. Then we have

Proposition 4 [M4, ACM]. Let $\nu>0$. Then $X_{G} \cup\{1\}=G$ if $G=H_{\tau}^{\nu}$, $\tau=0, \ldots, \nu+1$. Also, $X_{G} \cup\{1\}=G$ if $G=J_{1}$, where $J_{1}$ is the smallest Janko group.

$J_{1}$ is the only finite group $G$ known to satisfy $X_{G} \cup\{1\}=G$. Infinite groups $G$ satisfying $X_{G} \cup\{1\}=G$ were noted earlier (see e.g. [H]).

Fix an ordinal $\nu$. For any ordinal $\tau$ let $Y_{\nu}^{\tau}$ denote the set of all $\xi \in S_{\nu}^{\tau}$ satisfying (2), that is, $[\xi]^{2}$ contains a transposition. Obviously, Theorem 2 implies its generalization obtained by replacing " $\xi \in S$ " by " $\xi \in S_{\nu}$ ”.

Corollary 5. Let $\nu>0$, let $0 \leq \tau \leq \nu$, and let $\xi \in S_{\nu}^{\tau+1}$. The following are equivalent:

(a) $\xi \in X_{\nu}$.

(b) $\xi \in Y_{\nu}$ and $\xi$ moves $\aleph_{\tau}$ elements.

This corollary is established by Droste [D3, Corollary 3] for the case $0 \leq$ $\tau<\nu$ from the restricted formulation of Theorem 1 . Theorem 1 in full easily implies it for the case $0<\tau=\nu$, by the same argument.

The paper is organized as follows: In $\S 1$ we introduce some convenient notation, and use it to reformulate Theorems 1,2 and $\mathrm{A}$ as Theorems $1^{\prime}, 2^{\prime}$ and $\mathrm{A}^{\prime}$. We then prove Theorem $1^{\prime}$ using Theorem $2^{\prime}$, and Theorem $\mathrm{A}^{\prime}$. A proof of Theorem $2^{\prime}$ is also given there.

Theorem $A^{\prime}$, the main new result of this paper, is established in $\S \S 2-4$. In $\S 2$ we review the method of using bicolored planar maps. In $\S 3$ Theorem $A^{\prime}$ is reduced to two theorems, Theorem $\mathrm{B}$ and Theorem $\mathrm{C}$, whose proof relies on the use of bicolored planar maps.

Theorem B provides the inclusion claimed in Theorem A(1) and (2) for permutations $\zeta$ in INF-AI (in case (1)) or in INF (in case (2)) that have no infinite orbit, and is proved in $\S 3$.

Theorem $C$ takes care of the most difficult case-when $\zeta$ has at least one infinite orbit (where one easily sees that precisely one infinite orbit is the crucial case). It is proved in $\S 4$.

\section{Vocabulary}

In this section we introduce our notation and use it to reformulate our results.

$\mathbb{Z}$ denotes the set of integers, $\mathbb{N}$ denotes the set of positive integers, and $\mathbb{N}_{0}=\{0\} \cup \mathbb{N}, \mathbb{N}^{+}=\mathbb{N} \cup\left\{\aleph_{0}\right\} .|A|$ denotes the cardinality of the set $A$ and $S_{A}$ 
the group of all permutation of $A$. For $a \in A$ and $\xi \in S_{A}$ we let $\xi(a)$ denote the value taken by $\xi$ at $a$. Hence the composition $\xi \zeta$ is to be computed by acting first with $\zeta$, then with $\xi .1_{A} \in S_{A}$ is the identity permutation: $1_{A}(a)=a$ for all $a \in A$. If $B \subseteq A, \xi \mid B$ denotes the restriction of $\xi$ to $B$. If $\xi \mid B \in S_{B}$ then $B$ is called $\xi$-invariant. If $A=B_{1} \cup B_{2}, B_{1} \cap B_{2}=\varnothing$, $B_{1}$ and $B_{2}$ are $\xi$-invariant and $\xi_{i} \in S_{B_{i}}$ is $\xi \mid B_{i}, i=1,2$, then we write $\xi=\xi_{1}+\xi_{2}$. More generally, if $\left\{B_{i}: i \in I\right\}$ is a partition of $A$ into $\xi$-invariant sets, $\xi_{i}=\xi \mid B_{i}, i \in I$, then we write $\xi=\sum_{i \in I} \xi_{i}$.

For $\xi \in S_{A}$ and $a \in A$ let $(a)_{\xi}=\left\{\xi^{n}(a): n \in \mathbb{Z}\right\} .(a)_{\xi}$ is called the $\xi$-orbit of $a$. Let $(A)_{\xi}=\left\{(a)_{\xi}: a \in A\right\}$ be the partition of $A$ into $\xi$-orbits, and for $n \in \mathbb{N}^{+}$let $(A)_{\xi, n}=\left\{B \in(A)_{\xi}:|B|=n\right\}$. We define the cardinal number $\bar{\xi}(n)$ by

$$
\bar{\xi}(n)=\left|(A)_{\xi, n}\right|=\text { cardinality of the set of } \xi \text {-orbits of cardinality } n \text {. }
$$

By a type we mean a cardinal valued function $t$ defined on $\mathbb{N}^{+}$. Thus, for any permutation $\xi \in S_{A}$, $\bar{\xi}$ is a type. Moreover, for $\xi, \eta \in S_{A}$ we have $[\xi]=[\eta]$ iff $\bar{\xi}=\bar{\eta}$ (see e.g. [S]). For types $s, t$ and a cardinal number $k$ we define the types $s+t$ and $k t$ by $(s+t)(n)=s(n)+t(n)$ and $(k t)(n)=k \cdot t(n)$ $\left(n \in \mathbb{N}^{+}\right)$. More generally, if $t_{i}$ is a type for all $i \in I$, then a type $\sum_{i \in I} t_{i}$ is defined by

Obviously we have

$$
\left(\sum_{i \in I} t_{i}\right)(n)=\sum_{i \in I} t_{i}(n) \quad\left(n \in \mathbb{N}^{+}\right) .
$$

$$
\overline{\xi+\eta}=\bar{\xi}+\bar{\eta}, \overline{\sum_{i \in I} \xi_{i}}=\sum_{i \in I} \bar{\xi}_{i} .
$$

Given three types $r, s, t$ we write $P(r, s, t)$ for the following statement:

There is a set $A$ and $\xi, \eta, \zeta \in S_{A}$ such that $\bar{\xi}=r, \bar{\eta}=s$,

$$
\bar{\zeta}=t \text { and } \xi \eta \zeta=1_{A} \text {. }
$$

We have $[\xi] \subseteq[\eta][\zeta]$ iff $P(\bar{\xi}, \bar{\eta}, \bar{\zeta})$.

The main properties of $P$ are (see [M4]):

Symmetry. $P\left(t_{1}, t_{2}, t_{3}\right)$ iff $P\left(t_{\theta(1)}, t_{\theta(2)}, t_{\theta(3)}\right)$ for some $\theta \in S_{\{1,2,3\}}$, iff $P\left(t_{\theta(1)}, t_{\theta(2)}, t_{\theta(3)}\right)$ for all $\theta \in S_{\{1,2,3\}}$.

Superadditivity. If $P\left(r_{i}, s_{i}, t_{i}\right)$ for all $i \in I$, then

$$
P\left(\sum_{i \in I} r_{i}, \sum_{i \in I} s_{i}, \sum_{i \in I} t_{i}\right) .
$$

From superadditivity follows also

Homogeneity. $P(r, s, t)$ implies $P(k r, k s, k t)$ for any cardinal number $k$.

For $n \in \mathbb{N}^{+}$define the types $n^{*}, \tilde{n}$ by:

$$
n^{*}(n)=1, \quad n^{*}(m)=0 \quad \text { for } m \neq n, \quad \tilde{n}=\aleph_{0} \cdot n^{*} .
$$


Then for any type $t$ we have $t=\sum_{n \in \mathbb{N}^{+}} t(n) \cdot n^{*}$. Define also $|t|=$ $\sum_{n \in \mathbb{N}^{+}} t(n) \cdot n$. Then for $\xi \in S_{A}$ we have $|\bar{\xi}|=|A|$.

If $K \subseteq \mathbb{N}^{+}, t$ a type, we set $t[K]=\sum_{n \in K} t(n)$. We adopt the usual conventions for intervals in $\mathbb{N}^{+}$: if $k, l \in \mathbb{N}^{+}$then:

$$
\begin{aligned}
(k, l) & =\left\{n \in \mathbb{N}^{+}: k<n<l\right\}, & {[k, l) } & =\left\{n \in \mathbb{N}^{+}: k \leq n<l\right\}, \\
(k, l] & =\left\{n \in \mathbb{N}^{+}: k<n \leq l\right\}, & {[k, l] } & =\left\{n \in \mathbb{N}^{+}: k \leq n \leq l\right\} .
\end{aligned}
$$

Let $\xi \in S_{A}$. We define $M(\xi) \subseteq A$ by $M(\xi)=\{a \in A: \xi(a) \neq a\}$.

A permutation $\xi \in S_{A}$ is finitary if $M(\xi)$ is finite, and a type $t$ is finitary if $t=\bar{\xi}$ for some finitary $\xi$. So $t$ is finitary iff $t\left(\aleph_{0}\right)=0$ and $t\left[\left(1, \aleph_{0}\right)\right]<\aleph_{0}$.

Let $\mathbf{x}_{0}=\left\{\bar{\xi}: \xi \in X_{0}\right\}$. Thus, $t \in \mathbf{x}_{\mathbf{0}}$ iff $P(t, s, t)$ holds true for every type $s$ with $|s|=\aleph_{0}$.

We formulate Theorem 1, Theorem A and Theorem 2 as follows:

Theorem $\mathbf{1}^{\prime}$. Let $t$ be a type. The following are equivalent:

(i) $t \in \mathbf{x}_{\mathbf{0}}$.

(ii) $|t|=\aleph_{0}$ and $t$ satisfies one of the following three mutually exclusive conditions:

$\left(1^{\prime}\right) \quad t\left(\aleph_{0}\right) \geq 2$.

(2') (a) $t\left(\aleph_{0}\right)=1$.

(b) $t(n)=\aleph_{0}$ for some $n \in \mathbb{N}$.

(3') (a) $t\left(\aleph_{0}\right)=0$.

(b) $t(k)=t(l)=t(k+l)=\aleph_{0}$ for some $k, l \in \mathbb{N}$.

(c) $t\left[\left(2, \aleph_{0}\right)\right]=\aleph_{0}$.

Let inf $=\{\bar{\xi}: \xi \in \mathrm{INF}\}$. Thus $t \in$ inf iff $|t|=\aleph_{0}$ and $t\left(\aleph_{0}\right)>0$ or $t\left[\left(1, \aleph_{0}\right)\right]=\aleph_{0}$. Define ai $\subseteq$ inf by ai $=\{\bar{\xi}: \xi \in \mathrm{AI}\}$. Thus $t \in$ ai iff $t(2)=\aleph_{0}$, $t\left[\left(2, \aleph_{0}\right)\right]<\aleph_{0}$ and $t\left(\aleph_{0}\right)=0$.

Theorem $\mathbf{A}^{\prime}$. Let $r, t \in$ inf satisfy $r\left(\aleph_{0}\right)=t\left(\aleph_{0}\right)=0$.

(1) If $r\left[\left[3, \aleph_{0}\right)\right]=t\left[\left[3, \alpha_{0}\right)\right]=\aleph_{0}$ and $s \in$ inf-ai , then $P(r, s, t)$.

(2) If $r\left[\left[4, \aleph_{0}\right)\right]=t\left[\left[4, \aleph_{0}\right)\right]=\aleph_{0}$ and $s \in$ inf, then $P(r, s, t)$.

(3) Statement (1) fails if we require only $r\left[\left[2, \aleph_{0}\right)\right]=t\left[\left[2, \aleph_{0}\right)\right]=\aleph_{0}$. Statement (2) fails if we require only $r\left[\left[3, \aleph_{0}\right)\right]=t\left[\left[3, \aleph_{0}\right)\right]=\aleph_{0}$.

Let $\mathbf{y}_{\mathbf{0}}=\left\{\bar{\xi}: \xi \in Y_{0}\right\}$. Thus, $t \in \mathbf{y}_{\mathbf{0}}$ iff $P\left(t, \tilde{1}+2^{*}, t\right)$. Obviously, $\mathbf{x}_{\mathbf{0}} \subseteq \mathbf{y}_{\mathbf{0}}$.

Theorem $2^{\prime}$. Let $t$ be a type. Then the following are equivalent:

(i) $t \in \mathbf{y}_{\mathbf{0}}$.

(ii) $|t|=\aleph_{0}$ and $t$ satisfies one of the following three mutually exclusive conditions:

(1') $t\left(\aleph_{0}\right) \geq 2$.

(2') $t\left(\aleph_{0}\right)=1$ and $t(n)=\aleph_{0}$ for some $n \in \mathbb{N}$.

$\left(3^{\prime \prime}\right) \quad t\left(\aleph_{0}\right)=0$ and $t(k)=t(l)=t(k+l)=\aleph_{0}$ for some $k, l \in \mathbb{N}$. 
We now reformulate two earlier results and use them, together with Theorem $\mathrm{A}^{\prime}$ and Theorem $2^{\prime}$, to prove Theorem $1^{\prime}$. The first, due to Droste, is a restricted form of Theorem $1^{\prime}$.

Proposition 1.1 (Droste [D1, Theorem 1; D3, Theorem 2]). Let $t$ be a type, $|t|=\aleph_{0}$, and $t\left(\aleph_{0}\right) \geq 1$ or $t(1)=\aleph_{0}$. Then the following are equivalent:

(i) $t \in \mathbf{x}_{\mathbf{0}}$.

(ii) $t$ satisfies one of the three mutually exclusive conditions $\left(1^{\prime}\right),\left(2^{\prime}\right),\left(3^{\prime}\right)$ of Theorem $1^{\prime}$.

(The fact that if $t$ satisfies $\left(1^{\prime}\right)$ of Theorem $1^{\prime}$ then $t \in \mathbf{x}_{0}$ was noted already in 1981 by Droste and Göbel; see [DG2].)

Proposition 1.2 (see [M2, Corollary 2.5; D2, Theorem 4.1]). Let $t \in$ ai and let $P(t, s, t)$. Then $s\left[\mathbb{N}^{+}\right]=\aleph_{0}$. Hence $t \notin \mathbf{x}_{0}$, as $P\left(t, \aleph_{0}^{*}, t\right)$ does not hold, for instance.

Proof of Theorem $1^{\prime}$ (assuming Theorems $2^{\prime}$ and $\mathrm{A}^{\prime}$ ).

(i) $\rightarrow$ (ii) Let $t \in \mathbf{x}_{\mathbf{0}}$. By $\mathbf{x}_{\mathbf{0}} \subseteq \mathbf{y}_{0}, t \in \mathbf{y}_{0}$. By Theorem $2^{\prime}, t$ satisfies one of the conditions $\left(1^{\prime}\right),\left(2^{\prime}\right)$, or $\left(3^{\prime \prime}\right)$. But $\left(3^{\prime \prime}\right)$ is equivalent to $\left(3^{\prime}\right)($ a) and $\left(3^{\prime}\right)(b)$. Thus, it is left to show that if $t$ satisfies $\left(3^{\prime \prime}\right)$ it satisfies also $\left(3^{\prime}\right)(\mathrm{c})$, i.e., $t \notin$ ai. But $t \notin$ ai follows from Proposition 1.2.

(ii) $\rightarrow$ (i) Let $t$ satisfy (ii), i.e., $|t|=\aleph_{0}$ and $t$ satisfies $\left(1^{\prime}\right),\left(2^{\prime}\right)$ or $\left(3^{\prime}\right)$. If $t$ satisfies $\left(1^{\prime}\right)$ or $\left(2^{\prime}\right)$ then $t \in \mathbf{x}_{0}$ by Proposition 1.1. If $t$ satisfies $\left(3^{\prime}\right)$ and $t(1)=\aleph_{0}$ then again $t \in \mathbf{x}_{0}$ by Proposition 1.1, so assume $t$ satisfies $\left(3^{\prime}\right)$ but $t(1)<\aleph_{0}$. By $\left(3^{\prime}\right)(\mathrm{b}) t(k)=t(l)=t(k+l)=\aleph_{0}$ for some $k, l \in \mathbb{N}$, and by $t(1)<\aleph_{0}, 2 \leq k, 2 \leq l$ so $k+l \geq 4$. Thus, $t\left[\left[4, \aleph_{0}\right)\right]=\aleph_{0}$. By Theorem $\mathrm{A}^{\prime}(2)$ and [D3, Theorem 1], $t \in \mathbf{x}_{\mathbf{0}}$.

Proof of Theorem $2^{\prime}$. We first make the following observation: Let $A=B \cup B^{\prime}$, $B \cap B^{\prime}=\varnothing, b \in B$, and $b^{\prime} \in B^{\prime}$. Let $\xi \in S_{A}$ and let $B=(b)_{\xi}, B^{\prime}=\left(b^{\prime}\right)_{\xi}$ (so $B, B^{\prime}$ are $\xi$-orbits). Let $\left(b, b^{\prime}\right)$ be the transposition interchanging $b$ and $b^{\prime}$. Let $\zeta=\left(b, b^{\prime}\right) \xi$. Then we have:

(1) If $|B|=\left|B^{\prime}\right|=\aleph_{0}$ then $\bar{\zeta}=2 \cdot \aleph_{0}^{*}$, i.e. $\zeta$ has two infinite orbits.

(2) If $|B|=\aleph_{0}$ and $\left|B^{\prime}\right|=n<\aleph_{0}$ then $\bar{\zeta}=\aleph_{0}^{*}$, i.e. $\zeta$ has one infinite orbit.

(3) If $|B|=k$ and $\left|B^{\prime}\right|=l, k, l<\aleph_{0}$, then $\bar{\zeta}=:(k+l)^{*}$, i.e. $\zeta$ has one orbit, of size $k+l$.

Thus we have

(1) $P\left(2 \cdot \aleph_{0}^{*}, \tilde{1}+2^{*}, 2 \cdot \aleph_{0}^{*}\right)$.

(2) $P\left(\aleph_{0}^{*}, \tilde{1}+2^{*}, n^{*}+\aleph_{0}^{*}\right), n \in \mathbb{N}$.

(3) $P\left((k+l)^{*},(k+l-2) \cdot 1^{*}+2^{*}, k^{*}+l^{*}\right)$.

Also:

(4) $P\left(t,|t| \cdot 1^{*}, t\right)$ holds for any type $t$. 
(ii) $\rightarrow$ (i) Assume that $|t|=\aleph_{0}$ and $t$ satisfies $\left(1^{\prime}\right),\left(2^{\prime}\right)$ or $\left(3^{\prime \prime}\right)$. We show that $t \in \mathbf{y}_{\mathbf{0}}$, i.e., $P\left(t, \tilde{1}+2^{*}, t\right)$.

In case $\left(1^{\prime}\right)$ we have $t=2 \cdot \kappa_{0}^{*}+t^{\prime}$ so by (1) and (4) $P\left(t, \tilde{1}+2^{*}, t\right)$.

In case $\left(2^{\prime}\right)$ we have $t=\aleph_{0}^{*}+\tilde{n}+t^{\prime}=n^{*}+\kappa_{0}^{*}+\tilde{n}+t^{\prime}$, so by (2) and (4) $P\left(t, \tilde{\mathrm{l}}+2^{*}, t\right)$.

In case $\left(3^{\prime}\right)$ we have $t=\tilde{k}+\tilde{l}+(\widetilde{k+l})+t^{\prime}$, and so we have $t=(k+l)^{*}+t^{\prime}=$ $k^{*}+l^{*}+t^{\prime}$, and so by (3) and (4) $P\left(t, \tilde{1}+2^{*}, t\right)$.

(i) $\rightarrow$ (ii) Let $t \in \mathbf{y}_{\mathbf{0}}$. Then there are $\xi, \zeta \in S_{A}$ and a transposition $\left(b, b^{\prime}\right) \in$ $S_{A}$ such that $\zeta=\left(b, b^{\prime}\right) \xi$, and $\bar{\xi}=\bar{\zeta}=t$. We may further assume that $b$ and $b^{\prime}$ belong to distinct $\xi$-orbits $B$ and $B^{\prime}$ (else $b$ and $b^{\prime}$ belong to distinct $\zeta$-orbits and we have $\left.\xi=\left(b, b^{\prime}\right) \zeta\right)$. Let $\xi=\xi^{\prime}+\xi^{\prime \prime}, \zeta=\zeta^{\prime}+\zeta^{\prime \prime}$ where $\xi^{\prime}, \zeta^{\prime} \in S_{B \cup B^{\prime}}$. Then we have $\zeta^{\prime}=\left(b, b^{\prime}\right) \xi^{\prime}$ (where here $\left.\left(b, b^{\prime}\right) \in S_{B \cup B^{\prime}}\right)$, $\xi^{\prime \prime}=\left(\zeta^{\prime \prime}\right)^{-1}$. Hence $\bar{\xi}^{\prime}=|B|^{*}+\left|B^{\prime}\right|^{*}$, and $\bar{\xi}^{\prime \prime}=\bar{\zeta}^{\prime \prime}$. Now $t=\bar{\xi}^{\prime}+\bar{\xi}^{\prime \prime}=\bar{\zeta}^{\prime}+\bar{\zeta}^{\prime \prime}$, and $P\left(\bar{\xi}^{\prime},\left(\overline{b, b^{\prime}}\right), \bar{\zeta}^{\prime}\right)$ imply that $t$ satisfies $\left(1^{\prime}\right),\left(2^{\prime}\right)$ or $\left(3^{\prime \prime}\right)$ as follows.

We know that $\bar{\xi}^{\prime}=2 \cdot \aleph_{0}^{*}$, or $\bar{\xi}^{\prime}=\aleph_{0}^{*}+n^{*}$, or $\bar{\xi}^{\prime}=k^{*}+l^{*} \quad(n, k, l \in \mathbb{N})$. If $\bar{\xi}^{\prime}=2 \cdot \aleph_{0}^{*}$ then $t\left(\aleph_{0}\right) \geq 2$ so $\left(1^{\prime}\right)$ holds. If $\bar{\xi}^{\prime}=\aleph_{0}^{*}+n^{*}$ then $\bar{\zeta}^{\prime}=\aleph_{0}^{*}$ and so setting $t^{\prime \prime}=\bar{\xi}^{\prime \prime}=\bar{\zeta}^{\prime \prime}$ we have $t=\aleph_{0}^{*}+n^{*}+t^{\prime \prime}=\aleph_{0}^{*}+t^{\prime \prime}$, so $t\left(\aleph_{0}\right) \geq 1$, and by $t(n)=t^{\prime \prime}(n)+1=t^{\prime \prime}(n)$, we have $t(n)=\aleph_{0}$. Thus $t$ satisfies $\left(1^{\prime}\right)$ or $\left(2^{\prime}\right)$.

If $\bar{\xi}^{\prime}=k^{*}+l^{*}$ then $\bar{\zeta}^{\prime}=(k+l)^{*}$ and we obtain as before $t=k^{*}+l^{*}+$ $t^{\prime \prime}=(k+l)^{*}+t^{\prime \prime}$, so $t(k)=1+t^{\prime \prime}(k)=t^{\prime \prime}(k), t(l)=1+t^{\prime \prime}(l)=t^{\prime \prime}(l)$, $t(k+l)=1+t^{\prime \prime}(k+l)=t^{\prime \prime}(k+l)$.

It follows that $t(k), t(l), t(k+l) \geq \aleph_{0}$, and $t$ satisfy $\left(1^{\prime}\right),\left(2^{\prime}\right)$ or $\left(3^{\prime}\right)$.

\section{BICOLORED PLANAR MAPS REVISITED}

In this section we review briefly the bicolored-planar-map method introduced in [M4], and use it to establish Theorem $A^{\prime}$ in subsequent sections. The following treatment is self-contained and slightly different from the one offered in [M4].

By a planar graph we mean a pair $G=\left(V_{G}, E_{G}\right)$ where $V=V_{G}$ is a set of points in the plane, called the vertices of $G$, and $E=E_{G}$ is the set of edges of $G$. Each edge $e \in E$ is the range of a continuous mapping $f$ of the closed unit interval $[0,1]$ into the plane, whose restriction to the open unit interval $(0,1)$ is a homeomorphism. $f(0)$ and $f(1)$ are called the endpoints of $e$. They should be vertices, and may be nondistinct. If $f(0)=f(1)$ we call $e$ a loop. Two distinct edges may have the same set of endpoints (multiplicity of edges (including loops) is allowed). An edge $e$ meets $V$ by the set of its endpoints, and the intersection of two distinct edges is a set of vertices (so two edges meet at common endpoints). In addition to those usual conditions, we impose two more assumptions as follows:

(a) every bounded set meets only finitely many edges. 
The degree $d(v)$ of $v \in V$ is defined as the number of times $v$ occurs as an endpoint of an edge (so a loop contributes two to this count). It follows from (a) that every vertex has a finite degree.

We let $G_{*}=V \cup(\bigcup E)$ denote the set of all points of the plane in $V$ or in $e$ for some $e \in E$. Our second assumption is:

(b) $G_{*}$ is connected.

It follows from (a) that the complement $G_{*}^{c}$ of $G_{*}$ is open. A connected component of $G_{*}^{c}$ is called a $G$-region. It further follows from (b) that every $G$ region is simply connected, and so homeomorphic to an open disc. Let $F=F_{G}$ denote the set of all $G$-regions.

By a map we mean a triple $(V, E, F)$, where $G=(V, E)$ is a planar graph and $F=F_{G}$ is the set of $G$-regions.

By a bicolored planar map (bpm) we mean a quadruple $(V, E, B, W)$, where $(V, E, B \cup W)$ is a map, and for distinct $b, b^{\prime} \in B \quad\left(w, w^{\prime} \in W\right)$ we have $\operatorname{cl}(b) \cap \operatorname{cl}\left(b^{\prime}\right) \subseteq V \quad\left(\operatorname{cl}(w) \cap \operatorname{cl}\left(w^{\prime}\right) \subseteq V\right)$. Here $\operatorname{cl}(X)$ is the closure of the set $X$ in the plane. $B(W)$ is the set of black (white) regions of the map.

Let $(V, E, B, W)$ be a bpm, $e \in E$. Then there is a unique $b_{e} \in B$ and a unique $w_{e} \in W$ such that $e \in \operatorname{cl}\left(b_{e}\right) \cap \operatorname{cl}\left(w_{e}\right)$. For a region $f \in B \cup W$, let $F_{f}=\left\{e \in E: f=f_{e}\right\}$ be the set of edges in its closure, and let $s(f)=\left|E_{f}\right|$. We say that $f$ is of size $s(f)$.

We now use the orientation of the plane to define three permutations $\beta, v$, $\omega \in S_{E}$ as follows. Orient $e \in E$ by requiring that a traveller moving on $e$ as oriented sees $b_{e}$ to his left and $w_{e}$ to his right. (Thus, the boundary of a bounded $b \in B$ is oriented counterclockwise.) Define the tail $t(e)$, and that head $h(e)$, of $e \in E$ to be the endpoint travelled from and to by this traveller on $e$.

Let us say that $e^{\prime}$ follows $e$ around $b \in B \quad(w \in W)$ iff $b=b_{e}=b_{e^{\prime}}$ $\left(w=w_{e}=w_{e^{\prime}}\right)$ and $h(e)=t\left(e^{\prime}\right)$.

Let $v \in V$, and let $E_{v}$ denote the set of edges $e \in E$ satisfying $t_{e}=v$ (so $E_{v}$ is the set of edges emenating from $v$ ). By (a) $E_{v}$ is a finite set. Let $s(v)=\left|E_{v}\right|$. Thus, $s(v)$ the out-degree of $v$. We have $d(v)=2 s(v)$, as our orientation makes $(V, E)$ into an Eulerian graph. We say that $v$ is of size $s(v)$.

Let $e, e^{\prime} \in E_{v}$. We say that $e^{\prime}$ follows $e$ around $v$ if for any small enough circle $C$ centered at $v, P_{e^{\prime}}$ follows $P_{e}$ in the clockwise sense on $C$, where $P_{e}$ $\left(P_{e^{\prime}}\right)$ is the first point on $C$ met by a traveller on $e\left(e^{\prime}\right)$ moving from $t(e)=v$ to $h(e) \quad\left(t\left(e^{\prime}\right)=v\right.$ to $\left.h\left(e^{\prime}\right)\right)$.

Define $\beta, v$ and $\omega$ to be the black, vertex and white permutations respectively acting on the set of edges $E$. That is:

$$
\begin{gathered}
\beta(e)=e^{\prime} \quad \text { iff } \quad e^{\prime} \text { follows } e \text { around } b_{e}=b_{e^{\prime}}, \\
v(e)=e^{\prime} \text { iff } e^{\prime} \text { follows } e \text { around } t_{e}=t_{e^{\prime}}, \\
\omega(e)=e^{\prime} \quad \text { iff } \quad e^{\prime} \text { follows } e \text { around } w_{e}=w_{e^{\prime}} .
\end{gathered}
$$


One readily verifies:

Proposition 2.1. Let $(V, E, B, W)$ be a bicolored planar map. Then $\beta, v, \omega \in$ $S_{E}$ defined above satisfy:

(1) $\left\{E_{b}: b \in B\right\}$ is the partition of $E$ into $\beta$-orbits.

(2) $\left\{E_{v}: v \in V\right\}$ is the partition of $E$ into v-orbits.

(3) $\left\{E_{w}: w \in W\right\}$ is the partition of $E$ into $\omega$-orbits.

(4) $\beta=v \omega$; hence, $P(\bar{\beta}, \bar{v}, \bar{\omega})$.

(5) Let $G=\langle\beta, v\rangle=\langle v, \omega\rangle=\langle\omega, \beta\rangle$ be the group of permutations of $E$ generated by any two of the three permutations $\beta, v, \omega$. Then $G$ acts transitively on $E$.

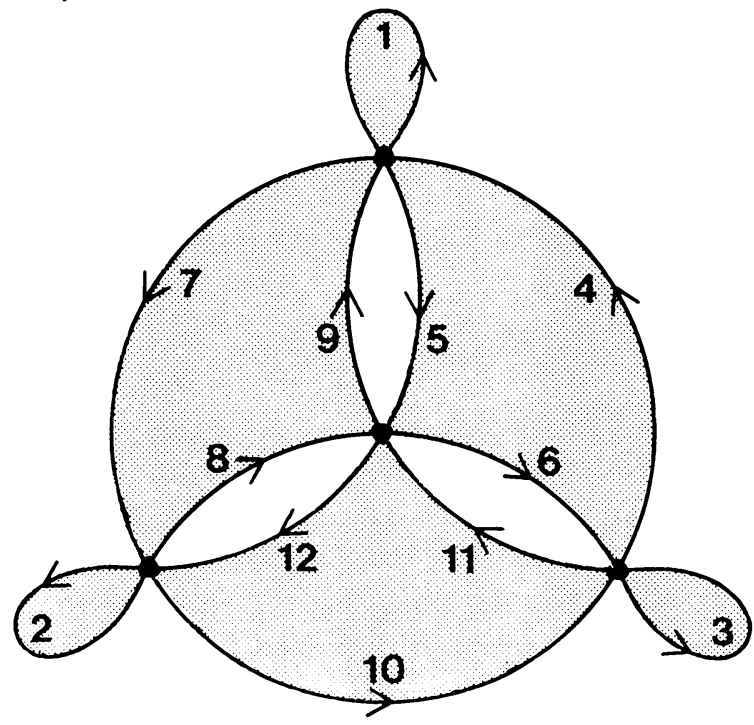

FIGURE 1

The edges of this bicolored planar map-marked here 1 to 12 -oriented as marked define the associated three permutations:

The black permutation $\beta$ :

$$
\beta=(1)(2)(3)(4,5,6)(7,8,9)(10,11,12)
$$

The vertex permutation $v$ :

$$
v=(1,5,7)(2,8,10)(3,11,4)(6,12,9)
$$

The white permutation $\omega$ :

$$
\omega=(1,7,2,10,3,4)(5,9)(6,11)(8,12)
$$

which satisfy $\beta=v \omega$.

The relation $P\left(3 \cdot\left(1^{*}+3^{*}\right), 4 \cdot 3^{*}, 6^{*}+3 \cdot 2^{*}\right)$ follows.

Figure 1 displays Proposition 2.1 in a particular case, where the bicolored map $M$ has 12 edges, oriented as required to define the permutations $\beta, v, \omega$.

Establishing $P$ relations on countable sets by means of bpms proved useful in the past. Here we shall use such maps to demonstrate that such relations can 
be established, while some extra side conditions are imposed. Figure 2 is an example. One concludes from it that permutations $\beta, v, \omega$ of a countable set exist such that:

(1) $\beta=v \omega$.

(2) $\bar{\beta}=\tilde{3}, \bar{v}=\tilde{1}+3 \cdot 2^{*}+\tilde{3}, \bar{\omega}=\tilde{5}$.

(3) Every $\omega$-orbit contains precisely one $v$-fixed point and $v$ has no other fixed points.

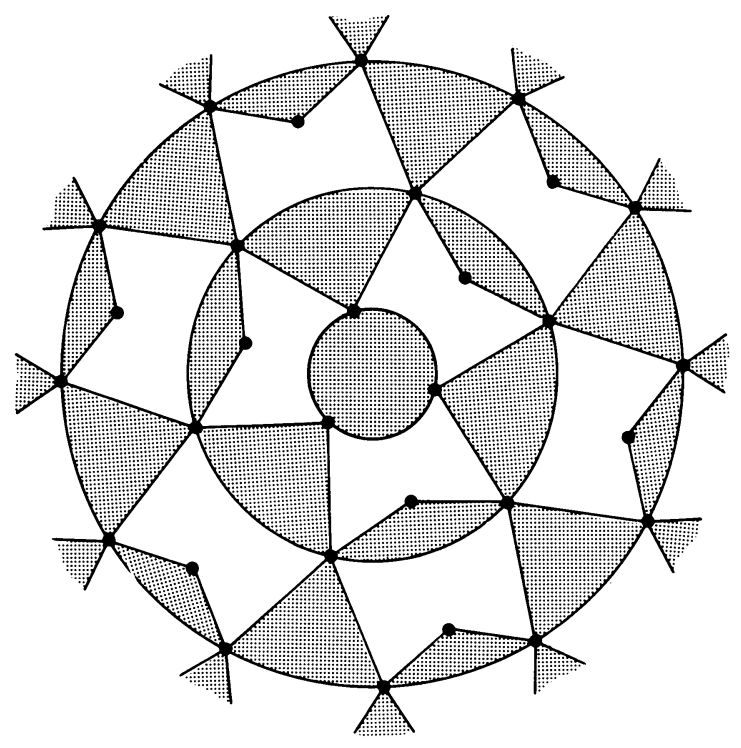

FIGURE 2

\section{Proof of Theorem A}

In this section we reduce the proof of Theorem $\mathrm{A}$ to the establishment of Theorem C, below. Theorem $\mathrm{C}$ is proved-using bicolored planar maps-in $\S 4$.

We first establish Theorem $\mathrm{A}(3)$, which asserts that in a sense Theorem $\mathrm{A}(1)$ and (2) is a sharp result.

Let $\xi, \eta \in \mathrm{INF}$ have no infinite orbit but infinitely many orbits of length at least $k$.

(a) If $k=2$, INF-AI $\subseteq[\xi] \cdot[\eta]$ does not necessarily follow.

Indeed, let $\xi=\eta$ be a fixed-point-free involution. Then $[\xi]=R_{0}$, and so $[\xi] \cdot[\eta]=R_{0}^{2}$ is the set of nicely even permutations, i.e. permutations $\zeta \in S$ with $\bar{\zeta}(n)$ an even cardinal for all $n \in \mathbb{N}^{+}$(see [M2]); and so, if $\zeta \in$ INF has a single infinite orbit, then $\zeta \notin R_{0}^{2}=[\xi] \cdot[\eta]$.

(b) If $k=3$, INF $\subseteq[\xi] \cdot[\eta]$ does not necessarily follow.

Indeed, if $\xi, \eta \in \mathrm{INF}$ satisfy $\xi^{3}=\eta^{3}=1$ and $[\xi] \neq[\eta]$, then $[\xi] \cdot[\eta]$ contains no involution (see [M5, Proposition 3.4]). (Even if $[\xi]=[\eta]$ then 
$[\xi][\eta]=[\xi]^{2}$ may omit some nonfinitary involutions; see [D3, Example 4.6].) Theorem $\mathrm{A}(3)$ is proved.

Theorem A(1) and (2) follow from the following two theorems.

Theorem B. Let $\xi, \eta \in \mathrm{INF}$ have no infinite orbit, but infinitely many orbits of length at least $k$, and let $\zeta \in \mathrm{INF}$ have no infinite orbit. Then:

(1) If $k=3$ and $\zeta$ has infinitely many orbits of length at least 3, then $\zeta \in[\xi][\eta]$.

(2) If $k=4$, then $\zeta \in[\xi][\eta]$.

Theorem C. Let $\xi, \eta \in \mathrm{INF}$ have no infinite orbit, but infinitely many orbits of length at least 3. Let $\zeta \in \mathrm{INF}$ have at least one infinite orbit. Then $\zeta \in[\xi] \cdot[\eta]$.

Let us restate these theorems, using the terminology developed in $\S 2$.

Theorem $\mathbf{B}^{\prime}$. Let $r, s, t$ be types satisfying

(i) $|r|=|s|=|t|=\aleph_{0}$.

(ii) $r\left(\aleph_{0}\right)=s\left(\aleph_{0}\right)=t\left(\aleph_{0}\right)=0$.

(iii) $r\left[\left[k, \aleph_{0}\right)\right]=s\left[\left[k, \aleph_{0}\right)\right]=t\left[\left[l, \aleph_{0}\right)\right]=\aleph_{0}$.

Then

(1) If $k=l=3$, then $P(r, s, t)$.

(2) If $k=4$ and $l=2$, then $P(r, s, t)$.

Theorem $\mathbf{C}^{\prime}$. Let $r, s, t$ be types satisfying:

(i) $|r|=|s|=\aleph_{0}$.

(ii) $r\left(\aleph_{0}\right)=s\left(\aleph_{0}\right)=0$.

(iii) $r\left[\left[3, \aleph_{0}\right)\right]=s\left[\left[3, \aleph_{0}\right)\right]=\aleph_{0}$.

(iv) $|t|=\aleph_{0}, t\left(\aleph_{0}\right) \geq 1$.

Then $P(r, s, t)$.

Theorem $C^{\prime}$ is the main technical result here, and will be proved in $\S 4$. The next two lemmas are special cases of Theorem $B^{\prime}$, from which Theorem $B^{\prime}$ easily follows.

Lemma B1. Let $m \in \mathbb{N}$, and let $r, s, t$ be types satisfying.

(i) $|r|=|s|=|t|=\aleph_{0}$.

(ii) $r\left(\aleph_{0}\right)=s\left(\aleph_{0}\right)=t\left(\aleph_{0}\right)=0$.

(iii) $r\left[\left[3, \aleph_{0}\right)\right]=s\left[\left[3, \aleph_{0}\right)\right]=t\left[\left[3, \aleph_{0}\right)\right]=\aleph_{0}$.

(iv) $r(1)=r(2)=s(1)=s(2)=t(1)=t(2)=0$.

Then $P\left(m^{*}+r, s, t\right), P\left(r, m^{*}+s, t\right), P\left(r, s, m^{*}+t\right)$.

Lemma B2. Let $m \in \mathbb{N}$, and let $r, s$ be types satisfying.

(i) $|r|=|s|=\aleph_{0}$.

(ii) $r\left(\aleph_{0}\right)=s\left(\aleph_{0}\right)=0$.

(iii) $r\left[\left[4, \aleph_{0}\right)\right]=s\left[\left[4, \aleph_{0}\right)\right]=\aleph_{0}$.

(iv) $r(1)=r(2)=r(3)=s(1)=s(2)=s(3)=0$. 
Then $P\left(m^{*}+r, s, \tilde{2}\right), P\left(r, m^{*}+s, \tilde{2}\right)$ and $P\left(r, s, m^{*}+\tilde{2}\right)$.

Proof of Theorem $\mathrm{B}^{\prime}$ from Lemmas $\mathrm{B} 1$ and $\mathrm{B} 2$.

Let $r, s, t$, be types satisfying (i)-(iii) of Theorem $\mathrm{B}^{\prime}$.

Case 1. Assume that $k=l=3$. By (iii) we can select three disjoint sets $I, J, K \subseteq \mathbb{N}$, and positive integers $l_{i}$ for $i \in I, m_{j}$ for $j \in J, n_{k}$ for $k \in K$; and types $r_{p}, s_{p}, t_{p}$ for $p \in I \cup J \cup K$ such that

(a) $r_{p}, s_{p}, t_{p}$ satisfy (i)-(iv) of Lemma B1 for $p \in I \cup J \cup K$.

(b)

$$
\begin{aligned}
& r=\sum_{i \in I}\left(l_{i}^{*}+r_{i}\right)+\sum_{j \in J} r_{j}+\sum_{k \in K} r_{k}, \\
& s=\sum_{i \in I} s_{i}+\sum_{j \in J}\left(m_{j}^{*}+s_{j}\right)+\sum_{k \in K} s_{k}, \\
& t=\sum_{i \in I} t_{i}+\sum_{j \in J} t_{j}+\sum_{k \in K}\left(n_{k}^{*}+t_{k}\right) .
\end{aligned}
$$

By Lemma B1 and (a), $P\left(l_{i}^{*}+r_{i}, s_{i}, t_{i}\right), P\left(r_{j}, m_{j}^{*}+s_{j}, t_{j}\right), P\left(r_{k}, s_{k}, n_{k}^{*}+t_{k}\right)$ hold for $i \in I, j \in J, k \in K$. Hence by superadditivity of $P, P(r, s, t)$ follows from (b).

Case 2. Assume now that $k=4$ and $l=2$. If $t(2)<\aleph_{0}$ then by (iii) $t\left[\left[3, \aleph_{0}\right)\right]=\aleph_{0}$ and so, $P(r, s, t)$ follows from Case 1.

If $t(2)=\aleph_{0}$ then we select again disjoint subset $I, J, K \subseteq \mathbb{N}$, positive integers $l_{i}, m_{j}, n_{k}$ for $i \in I, j \in J, k \in K$ and types $r_{p}, s_{p}$, for $p \in I \cup J \cup K$ so that:

(a) $r_{p}, s_{p}$ satisfy (i)-(iv) of Lemma B2.

(b)

$$
\begin{aligned}
& r=\sum_{i \in I}\left(l_{i}^{*}+r_{i}\right)+\sum_{j \in J} r_{j}+\sum_{k \in K} r_{k}, \\
& s=\sum_{i \in I} s_{i}+\sum_{j \in J}\left(m_{j}^{*}+s_{j}\right)+\sum_{k \in K} s_{k}, \\
& t=|I| \cdot \tilde{2}+|J| \cdot \tilde{2}+\sum_{k \in K}\left(n_{k}^{*}+\tilde{2}\right) .
\end{aligned}
$$

By Lemma B2, (a), (b) and the superadditivity of $P, P(r, s, t)$ follows.

Lemmas B1 and B2 are proved by constructing an appropriate bicolored planar map and using Proposition 2.1. In fact, they follow from the following proposition on planar bicolored maps, which only slightly generalizes Proposition 5.1 of [M4]. (By the symmetry of $P$ and the conditions of these lemmas, only three $P$-relations need to be verified.)

Proposition B. Let $m \in \mathbb{N}$ and let $\left(l_{i}\right)_{i \in \mathbb{N}},\left(m_{j}\right)_{j \in \mathbb{N}}$ and $\left(n_{k}\right)_{k \in \mathbb{N}}$ be sequences of integers, satisfying at least one of the following three conditions:

(1) $m_{1}=m ; 2<l_{i}, m_{j}, n_{k}$ for $i, j, k \in \mathbb{N}, j>1$.

(2) $m_{1}=m ; 3<l_{i}, n_{k}, 1<m_{j}$ for $i, j, k \in \mathbb{N}, j>1$.

(3) $l_{1}=m ; 3<l_{i}, n_{k}, 1<m_{j}$ for $i, j, k \in \mathbb{N}, i>1$. 
Then there is a bicolored planar map $M$ and enumeration $\left(b_{i}\right)_{i \in \mathbb{N}},\left(v_{j}\right)_{j \in \mathbb{N}}$, $\left(w_{k}\right)_{k \in \mathbb{N}}$ of its black regions, vertices and white regions respectively, so that $\left|E_{b_{i}}\right|=l_{i},\left|E_{v_{j}}\right|=m_{j},\left|E_{w_{k}}\right|=n_{k}$ for $i, j, k \in \mathbb{N}$.

Hence $P\left(\sum_{i \in \mathbb{N}} l_{i}^{*}, \sum_{i \in \mathbb{N}} m_{i}^{*}, \sum_{i \in \mathbb{N}} n_{i}^{*}\right)$ holds, by Proposition 2.1.

(Recall that $E_{b_{i}}\left(E_{w_{k}}\right)$ is the set of edges in the closure of the black region $b_{i}$ (the white region $w_{k}$ ) and $2\left|E_{v_{i}}\right|$ is the degree of $v_{i}$.)

Proof. See Proposition 5.1 in [M4], which is Proposition B for $m=1$, and cases (1), (3). To obtain a proof for Proposition B in general we need only to modify the base of inductive construction of the bicolored map given there. In cases (1) and (2), the base consists of the unit disc, partitioned radially into $m$ white and $m$ black alternating regions, sharing the origin as a common vertex (Figure 3(a)), while in case (3) it consists of the unit disc as a black region, with $m$ vertices on its boundary (Figure $3(b)$ ).

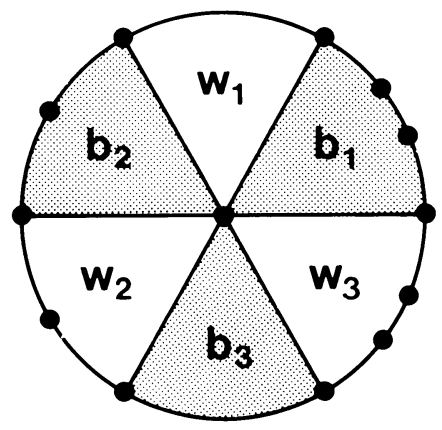

(a)

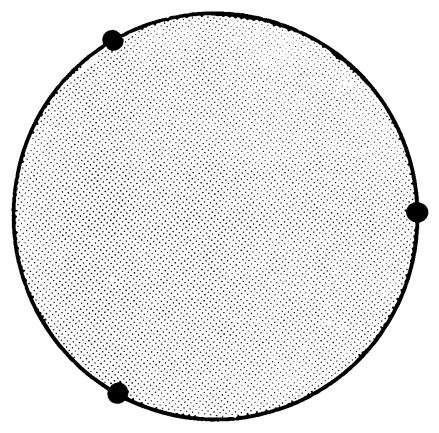

(b)

Figure 3. Base of induction for proof of Proposition B.

(a) Cases (1), (2), with: $m=3,\left(l_{1}, l_{2}, l_{3}\right)=(5,4,3)$, $\left(n_{1}, n_{2}, n_{3}\right)=(3,4,5)$.

(b) Case (3), with $m=3$.

\section{Proof of Theorem $C^{\prime}$}

First notice that we may assume in Theorem $C$ that $\zeta$ has precisely one infinite orbit, i.e., enough to prove the following special case of Theorem $C^{\prime}$.

Theorem $\mathbf{C}^{\prime \prime}$. Let $r, s, t$, be types satisfying.

(i) $|r|=|s|=\aleph_{0}$.

(ii) $r\left(\aleph_{0}\right)=s\left(\aleph_{0}\right)=0$.

(iii) $r\left[\left[3, \aleph_{0}\right)\right]=s\left[\left[3, \aleph_{0}\right)\right]=\aleph_{0}$.

(iv) $|t|=\aleph_{0}, t\left(\aleph_{0}\right)=1$.

Then $P(r, s, t)$. 
Indeed, assume Theorem $\mathrm{C}^{\prime \prime}$ and let $r, s, t$ satisfy assumptions (i)-(iv) of Theorern $\mathrm{C}^{\prime}$, with $t\left(\aleph_{0}\right)=k \geq 1$. Let $K \subseteq \mathbb{N}$ be a set of cardinality $k$. Then one can associate with each $i \in K$ types $r_{i}, s_{i}, t_{i}$ satisfying (i)-(iv) of Theorem $\mathrm{C}^{\prime \prime}$ and $r=\sum_{i \in K} r_{i}, s=\sum_{i \in K} s_{i}, t=\sum_{i \in K} t_{i}$. By Theorem $\mathrm{C}^{\prime \prime}, P\left(r_{i}, s_{i}, t_{i}\right)$ holds for each $i \in K$, and so by superadditivity of $P, P(r, s, t)$ holds as well.

The rest of this section is devoted to the proof of Theorem $C^{\prime \prime}$. We assume that $r, s, t$ are types satisfying (i)-(iv). In particular, $t=\aleph_{0}^{*}+t^{\prime \prime}$, where $t^{\prime \prime}\left(\aleph_{0}\right)=0$. Let $A$ be a set of cardinality $\aleph_{0}$. Our goal is to define $\xi, \eta, \zeta \in S_{A}$ such that $\bar{\xi}=r, \bar{\eta}=s, \bar{\zeta}=t$ and $\xi=\eta \zeta$. This is achieved in four steps as follows.

Step 1. Select types $r^{\prime}, r^{\prime \prime}, s^{\prime}, s^{\prime \prime}, t^{\prime}, t^{\prime \prime}$ so that:

(1.1) $r=r^{\prime}+r^{\prime \prime}, s=s^{\prime}+s^{\prime \prime}, t=t^{\prime}+t^{\prime \prime}$.

(1.2) $r^{\prime}\left[\left[3, \aleph_{0}\right)\right]=r^{\prime \prime}\left[\left[3, \aleph_{0}\right)\right]=s^{\prime}\left[\left[3, \aleph_{0}\right)\right]=s^{\prime \prime}\left[\left[3, \aleph_{0}\right)\right]=\aleph_{0}$.

(1.3) $t^{\prime}=\aleph_{0}^{*}$.

Let $A_{1}, A_{2}, A_{3}, A_{4} \subseteq A$ satisfy:

(1.4) $A=\bigcup_{i=1}^{4} A_{i}, A_{i} \cap A_{j}=\varnothing$ for $1 \leq i<j \leq 4$.

(1.5) $\left|A_{1}\right|=\left|A_{2}\right|=\left|A_{3}\right|=\aleph_{0},\left|A_{4}\right|=\left|t^{\prime \prime}\right|$.

We let $A_{i, j}=A_{i} \cup A_{j}$ and $A_{i j k}=A_{i} \cup A_{j} \cup A_{k}, 1 \leq i, j, k \leq 4$. Also, let $1_{i}=1_{A_{i}}, 1_{i j}=1_{A_{l j}}, 1_{l j k}=1_{A_{i j k}}, 1 \leq i, j, k \leq 4$.

Step 2. Define $\xi^{\prime} \in S_{A_{1}}, \eta^{\prime} \in S_{A_{12}}$, $\zeta^{\prime} \in S_{A_{123}}, \theta^{\prime} \in A_{23}$ so that:

(2.1) $\overline{\xi^{\prime}}=r^{\prime}, \overline{\eta^{\prime}}=s^{\prime}, \overline{\zeta^{\prime}}=\aleph_{0}^{*}, \overline{\theta^{\prime}}=\tilde{7}$.

(2.2) $\xi^{\prime}+\theta^{\prime}=\left(\eta^{\prime}+1_{3}\right) \zeta^{\prime}$.

Step 3. Define $\xi^{\prime \prime} \in S_{A_{234}}, \eta^{\prime \prime} \in S_{A_{34}}, \zeta^{\prime \prime} \in S_{A_{4}}$ so that:

(3.1) $\overline{\xi^{\prime \prime}}=r^{\prime \prime}, \overline{\eta^{\prime \prime}}=s^{\prime \prime}, \overline{\zeta^{\prime \prime}}=t^{\prime \prime}$.

(3.2) $\xi^{\prime \prime}=\left(1_{2}+\eta^{\prime \prime}\right)\left(\theta^{\prime}+\zeta^{\prime \prime}\right)$.

Step 4. Let $\xi=\xi^{\prime}+\xi^{\prime \prime}, \eta=\eta^{\prime}+\eta^{\prime \prime}, \zeta=\zeta^{\prime}+\zeta^{\prime \prime}$. Then

(4.1) $\bar{\xi}=r, \bar{\eta}=s, \bar{\zeta}=t$.

(4.2) $\xi=\eta \zeta$.

Theorem $C^{\prime \prime}$ follows from (4.1) and (4.2).

Details. Step 1 is obviously possible, and needs no further comment. Step 4 follows from the previous steps as follows: (4.1) follows from (1.1), (2.1) and 
(3.1). We establish (4.2):

$$
\begin{aligned}
\eta \zeta & =\left(\eta^{\prime \prime}+\eta^{\prime}\right)\left(\zeta^{\prime}+\zeta^{\prime \prime}\right)=\left[\left(1_{12}+\eta^{\prime \prime}\right)\left(\eta^{\prime}+1_{34}\right)\right]\left[\left(\zeta^{\prime}+1_{4}\right)\left(1_{123}+\zeta^{\prime \prime}\right)\right] \\
& =\left(1_{12}+\eta^{\prime \prime}\right)\left[\left(\left(\eta^{\prime}+1_{3}\right)+1_{4}\right)\left(\zeta^{\prime}+1_{4}\right)\right]\left(1_{123}+\zeta^{\prime \prime}\right) \\
& =\left(1_{12}+\eta^{\prime \prime}\right)\left[\left(\left(\eta^{\prime}+1_{3}\right) \zeta^{\prime}+1_{4} 1_{4}\right)\right]\left(1_{123}+\zeta^{\prime \prime}\right) \\
& =\left[\left(1_{1}+1_{2}+\eta^{\prime \prime}\right)\left(\xi^{\prime}+\theta^{\prime}+1_{4}\right)\right]\left(1_{1}+1_{23}+\zeta^{\prime \prime}\right) \\
& =\left(1_{1} \xi^{\prime}+\left(1_{2}+\eta^{\prime \prime}\right)\left(\theta^{\prime}+1_{4}\right)\right)\left(1_{1}+1_{23}+\zeta^{\prime \prime}\right) \\
& =1_{1} \xi^{\prime} 1_{1}+\left(1_{2}+\eta^{\prime \prime}\right)\left[\left(\theta^{\prime}+1_{4}\right)\left(1_{23}+\zeta^{\prime \prime}\right)\right] \\
& =\xi^{\prime}+\left(1_{2}+\eta^{\prime \prime}\right)\left(\theta^{\prime}+\zeta^{\prime \prime}\right)=\xi^{\prime}+\xi^{\prime \prime}=\xi
\end{aligned}
$$

Here we used (2.2) for the fifth equality and (3.2) for the last-but-one equality.

Steps 2 and 3 form the core of the argument, and follow from two slightly more general Propositions 4.1 and 4.2 that we formulate next.

Proposition 4.1. Let $r^{+}, s^{+}, t^{+}$be types satisfying:

(i) $\left|r^{+}\right|=\left|s^{+}\right|=\left|t^{+}\right|=\aleph_{0}$.

(ii) $r^{+}\left(\aleph_{0}\right)=s^{+}\left(\aleph_{0}\right)=t^{+}\left(\aleph_{0}\right)=0$.

(iii) $r^{+}\left[\left[3, \aleph_{0}\right)\right]=s^{+}\left[\left[2, \aleph_{0}\right)\right]=t^{+}\left[\left[2, \aleph_{0}\right)\right]=\aleph_{0}$.

Then there are $\xi^{\prime} \in S_{A_{1}}, \eta^{\prime} \in S_{A_{12}}, \zeta^{\prime} \in S_{A_{123}}, \theta^{\prime} \in S_{A_{23}}$ such that:

(4.1.1) $\overline{\xi^{\prime}}=r^{+}, \overline{\eta^{\prime}}=s^{+}, \overline{\zeta^{\prime}}=\aleph_{0}^{*}, \overline{\theta^{\prime}}=t^{+}$.

(4.1.2) $\xi^{\prime}+\theta^{\prime}=\left(\eta^{\prime}+1_{3}\right) \zeta^{\prime}$.

(4.1.3) If $D$ is any $\theta^{\prime}$-orbit then $D \cap A_{2}$ contains a single element $a_{D}$ (hence $A_{2}=\left\{a_{D}: D\right.$ is a $\theta^{\prime}$-orbit $\}$ ).

Proposition 4.2. Let $r^{++}, s^{++}, t_{0}^{++}, t_{1}^{++}$be types satisfying:

(i) $\left|r^{++}\right|=\left|s^{++}\right|=\left|t_{0}^{++}\right|=\aleph_{0},\left|t_{1}^{++}\right|=\left|A_{4}\right| \leq \aleph_{0}$.

(ii) $r^{++}\left(\aleph_{0}\right)=s^{++}\left(\aleph_{0}\right)=t_{0}^{++}\left(\aleph_{0}\right)=t_{1}^{++}\left(\aleph_{0}\right)=0$.

(iii) $r^{++}\left[\left[3, \aleph_{0}\right)\right]=s^{++}\left[\left[3, \aleph_{0}\right)\right]=t_{0}^{++}\left[\left[7, \aleph_{0}\right)\right]=\aleph_{0}, t_{0}^{++}[[1,6]]=0$.

Let further $\theta^{\prime} \in S_{A_{23}}$ be given so that:

(iv) $\overline{\theta^{\prime}}=t_{0}^{++}$.

(v) If $D$ is any $\theta^{\prime}$-orbit then $D \cap A_{2}$ contains a single element $a_{D}$ (hence $A_{2}=\left\{a_{D}: D\right.$ is a $\theta^{\prime}$-orbit $\}$ ).

Then there are $\xi^{\prime \prime} \in S_{A_{234}}, \eta^{\prime \prime} \in S_{A_{34}}$, $\zeta^{\prime \prime} \in S_{A_{4}}$ such that

$$
\begin{aligned}
& \overline{\xi^{\prime \prime}}=r^{++}, \overline{\eta^{\prime \prime}}=s^{++}, \overline{\zeta^{\prime \prime}}=t_{1}^{++} . \\
& \xi^{\prime \prime}=\left(1_{2}+\eta^{\prime \prime}\right)\left(\theta^{\prime}+\zeta^{\prime \prime}\right) .
\end{aligned}
$$

Let $M=(V, E, B, W)$ be a bicolored planar map. For $B^{\prime} \subseteq B, V^{\prime} \subseteq$ $V, W^{\prime} \subseteq W$, the types $t_{B^{\prime}}=t_{B^{\prime}}^{M}, t_{V^{\prime}}=t_{V^{\prime}}^{M}, t_{W^{\prime}}=t_{W^{\prime}}^{M}$ are defined as follows. 
Let $n \in \mathbb{N}^{+}$. Then

$$
\begin{aligned}
& t_{B^{\prime}}(n)=\text { number of black regions } b \in B^{\prime} \text { of size } n, \\
& t_{V^{\prime}}(n)=\text { number of vertices } v \in V^{\prime} \text { of size } n, \\
& t_{W^{\prime}}(n)=\text { number of white regions } w \in W^{\prime} \text { of size } n .
\end{aligned}
$$

(Recall that the size of a region $f$ is $\left|E_{f}\right|$, where $E_{f}$ is the set of edges in its closure, and the size of a vertex $v$ is $\left|E_{v}\right|$, which equals half its degree.) $t_{B}$ $\left(t_{V}, t_{W}\right)$ is called the black (the vertex, the white) type of $M$.

Propositions 4.1 and 4.2 follow from their respective counterparts, Propositions $4.1^{\prime}$ and $4.2^{\prime}$, that provide the necessary bicolored maps, using Proposition 2.1. We start with:

Proposition 4.1' . Let $r^{+}, s^{+}, t^{+}$be types satisfying (i)-(iii) of Proposition 4.1. Then there is a bicolored planar map $M=(V, E, B, W)$, and partitions $\left\{E_{1}, E_{2}, E_{3}\right\}$ of $E,\left\{B^{\prime}, B^{\prime \prime}\right\}$ of $B,\left\{V^{\prime}, V^{\prime \prime}\right\}$ of $V$ such that: $\bigcup_{v \in V^{\prime \prime}} E_{v}$.

(4.1'.1) $E_{1}=\bigcup_{b \in B^{\prime}} E_{b}, E_{2} \cup E_{3}=\bigcup_{b \in B^{\prime \prime}} E_{b}, E_{1} \cup E_{2}=\bigcup_{v \in V^{\prime}} E_{v}, E_{3}=$

(4.1'.2) $t_{B^{\prime}}=r^{+}, t_{B^{\prime \prime}}=t^{+}, t_{V^{\prime}}=s^{+}, t_{V^{\prime \prime}}=\tilde{1}, t_{W}=\kappa_{0}^{*}$.

(4.1'.3) If $b \in B^{\prime \prime}$ then $E_{b} \cap E_{2}$ contains a single element.

Proof of Proposition 4.1 from 4.1' . Let $\delta: E \rightarrow A_{123}$ be any bijection that maps $E_{i}$ onto $A_{i}, i=1,2,3$. Let $\beta, v$ and $\omega$ be the black, vertex and white permutations of $M$. Let $\beta^{\prime}=\beta\left|E_{1}, \beta^{\prime \prime}=\beta\right| E_{2} \cup E_{3}, v^{\prime}=v \mid E_{1} \cup E_{2}, v^{\prime \prime}=$ $v \mid E_{3}$, and define $\xi^{\prime}, \eta^{\prime}, \zeta^{\prime}, \theta^{\prime}$ by $\xi^{\prime}=\delta \beta^{\prime} \delta^{-1}, \eta^{\prime}=\delta v^{\prime} \delta^{-1}, \zeta^{\prime}=\delta \omega \delta^{-1}, \theta^{\prime}=$ $\delta \beta^{\prime \prime} \delta^{-1}$. Then (4.1.1)-(4.1.3) hold. (See Proposition 2.1 for (4.1.2).)

Proof of Proposition 4.1' - The Caterpillar Map. A bicolored planar map $M=$ $(V, E, B, W)$ is called a one way infinite caterpillar map-or, briefly, a caterpillar map-if it satisfies the following conditions:

All black regions have finite size (hence are bounded) and the set $B$ of black regions is a disjoint union $B=B^{+} \cup B^{++}$where:

(C.1) $B^{+}=\left\{b_{n}: n \in \mathbb{N}\right\}$ where $\operatorname{cl}\left(b_{n}\right) \cap \operatorname{cl}\left(b_{m}\right)=\varnothing$ if $|n-m|>1$, while $\operatorname{cl}\left(b_{n}\right) \cap \operatorname{cl}\left(b_{n+1}\right)=\left\{v_{n}\right\}, v_{n} \in V \quad(n \in \mathbb{N})$. The regions $b_{n} \in B^{+}$are called links and the vertices $v_{n}$ are called joints. Other vertices of $b_{n}$ are called nonjoints.

(C.2) Every $b^{\prime \prime} \in B^{++}$has a unique vertex $v_{b^{\prime \prime}}$ such that $\operatorname{cl}\left(b^{\prime \prime}\right) \cap \operatorname{cl}\left(b_{n}\right)=$ $\left\{v_{b^{\prime \prime}}\right\}$ for some $n \in \mathbb{N}\left(v_{b^{\prime \prime}}\right.$ may be a joint or a nonjoint $)$. The regions $b^{\prime \prime} \in$ $B^{++}$are called toes.

It follows that $W=\{w\}$, i.e. there is only one (unbounded) white region.

Assume now that $r^{+}, s^{+}, t^{+}$are types satisfying (i)-(iii) of Proposition 4.1. We shall show that a caterpillar map $M=(V, E, B, W)$ exists with partitions $\left\{E_{1}, E_{2}, E_{3}\right\}$ of $E,\left\{B^{\prime}, B^{\prime \prime}\right\}$ of $B$ and $\left\{V^{\prime}, V^{\prime \prime}\right\}$ of $V$ such that (4.1'.1)(4.1'.3) hold.

(a) Let $\left\{b_{n}: n \in \mathbb{N}\right\}$ be a sequence of disjoint translation of the unit disc in the plane such that (C.1) holds. Let $C_{n}$ denote the boundary of $b_{n}$ and let $v_{n}$ be the point of intersection of $C_{n}$ with $C_{n+1}$. 


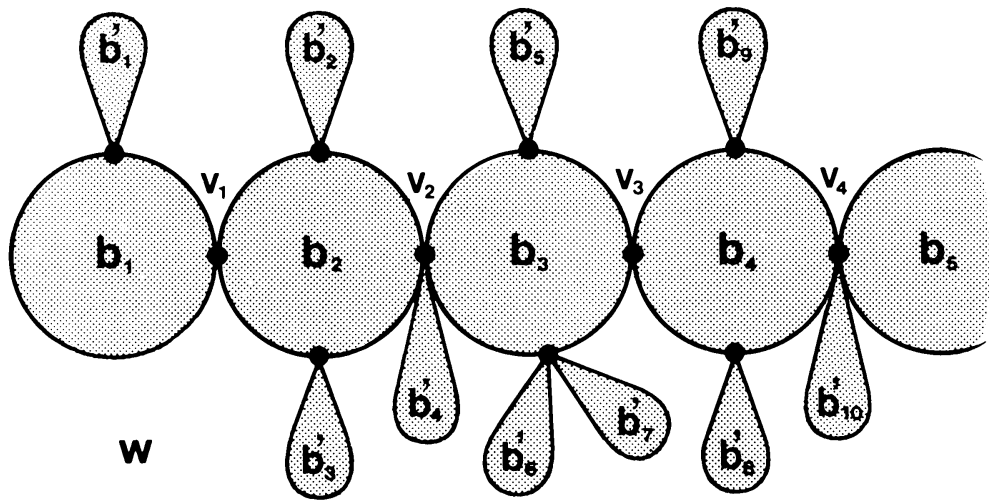

FIGURE 4. A caterpillar map

The black faces $b_{1}, \ldots, b_{5}, \ldots$ are links.

The black faces $b_{1}^{\prime}, \ldots, b_{10}^{\prime}$, are toes.

The vertices $v_{1}, \ldots, v_{4}, \ldots$ are joints.

The infinite region $w$ is the only white face.

(b) Let $\left(r_{n}\right)_{n \in \mathbb{N}}$ be a sequence of integers satisfying $r_{n}>1$ and $r^{+}=$ $r^{+}(1) \cdot 1^{*}+\sum_{n \in \mathbb{N}} r_{n}^{*}$. Make $b_{n}$ into a black region of size $r_{n}$ by adding, if necessary, extra vertices on its boundary $C_{n}$. Notice that, for $n>1, b_{n}$ already has $v_{n-1}$ and $v_{n}$ as vertices in its closure. We add more vertices if $r_{n}>2$, and this is true of infinitely many $n$ 's by $r^{+}\left[\left[3, \aleph_{0}\right)\right]=\aleph_{0}$. Let $B_{0}^{\prime}=\left\{b_{n}: n \in \mathbb{N}\right\}$ be the set of links of $M$.

(c) Let $\left(s_{k}\right)_{k \in \mathbb{N}}$ be a sequence of integers satisfying $s_{k}>0$ and $s^{+}=$ $\sum_{k \in \mathbb{N}} s_{k}^{*}$. Let $\left(u_{k}\right)_{k \in \mathbb{N}}$ be an enumeration of all vertices defined so far, such that:

1. If $s_{k}=1$ then $u_{k}$ is not a joint.

2. $s_{k}>1$ for infinitely many nonjoint vertices $u_{k}$.

(d) Add loops lying in the unbounded region, each enclosing a black region and pending at a vertex $u_{k}$ as necessary to guarantee that the degree of $u_{k}$ is $2 s_{k}$ (and so its size is $s_{k}$ ). Select a coinfinite subset $B_{1}^{\prime}$ of black regions enclosed by these loops of cardinality $\left|B_{1}\right|=r^{+}(1)$, and set $B^{\prime}=B_{0}^{\prime} \cup B_{1}^{\prime}$ (so $\left.t_{B^{\prime}}=r^{+}\right)$. Let $\left(b_{l}^{\prime \prime}\right)_{l \in \mathbb{N}}$ be an enumeration of the black regions enclosed by the other loops.

(e) Let $\left(t_{l}\right)_{l \in \mathbb{N}}$ be a sequence of integers satisfying $t^{+}=\sum_{l \in \mathbb{N}} t_{l}^{*}$. Add vertices on the loop enclosing $b_{l}^{\prime \prime}$ so as to make the size of $b_{l}^{\prime \prime}$ equal $t_{l}$. Notice that by $t^{+}[[2, \infty)]=\aleph_{0}, t_{l}>1$ for infinitely many l's. Let $B^{\prime \prime}=\left\{b_{l}^{\prime \prime}: l \in \mathbb{N}\right\}$ (so $t_{B^{\prime \prime}}=t^{+}$).

The caterpillar map $M=(V, E, B, W)$ is now complete. (Its "body" consists of the links $b_{n}$ of $B_{0}^{\prime}$; its "toes" form $B_{1}^{\prime} \cup B^{\prime \prime}$.) We let $V^{\prime}=\left\{u_{k}: k \in \mathbb{N}\right\}$ be the set of vertices that lie is the closure of some link $b^{\prime} \in B^{\prime}$ and $V^{\prime \prime}=$ 
$V-V^{\prime}$. Then $E_{1}, E_{2}, E_{3}$ are defined by $\left(4.1^{\prime} .1\right)$. A straightforward checking verifies $\left(4.1^{\prime} .1\right)-\left(4.1^{\prime} .3\right)$.

Proposition 4.2 . Let $r^{++}, s^{++}, t_{0}^{++}, t_{1}^{++}$be types satisfying (i)-(iii) of Proposition 4.2. Then there is a bicolored planar map $M=(V, E, B, W)$ and partitions $\left\{E_{2}, E_{3}, E_{4}\right\}$ of $E,\left\{V^{\prime}, V^{\prime \prime}\right\}$ of $V,\left\{W^{\prime}, W^{\prime \prime}\right\}$ of $W$ such that:

(4.2'.1) $E_{2} \cup E_{3}=\bigcup_{w \in W^{\prime}} E_{w}, E_{4}=\bigcup_{w \in W^{\prime \prime}} E_{w}, E_{3} \cup E_{4}=\bigcup_{v \in V^{\prime}} E_{v}$, $E_{2}=\bigcup_{v \in V^{\prime \prime}} E_{v}$.

(4.2 $\left.2^{\prime} .2\right) t_{B}=r^{++}, t_{V^{\prime}}=s^{++}, t_{V^{\prime \prime}}=\tilde{1}, t_{W^{\prime}}=t_{0}^{++}, t_{W^{\prime \prime}}=t_{1}^{++}$.

$\left(4.2^{\prime} .3\right)$ If $w \in W^{\prime}$ then $\mathrm{cl}(w)$ contains a unique distinguished vertex $v_{w} \in$ $V^{\prime \prime}$ of degree 2.

(4.2'.4) Let $w \in W^{\prime}$ and let $E_{v_{w}}=\left\{e_{w}\right\} \cdot{ }^{1}$ Then $E_{v_{w}}=E_{w} \cap E_{2}$ (hence $\left.E_{2}=\left\{e_{w}: w \in W^{\prime}\right\}\right)$.

Proof of Proposition 4.2 from 4.2' . Let $M$ be the map provided by Proposition $4.2^{\prime}$, and let $\beta, v, \omega \in S_{E}$ be its black, vertex and white permutations, respectively (see $\S 2$ ). Let $\theta^{\prime} \in S_{A_{23}}$ satisfy (iv) and (v) of Proposition 4.2. Since $\overline{\theta^{\prime}}=t_{0}^{++}=t_{W^{\prime}}$ there is a bijection $\varepsilon$ from $W^{\prime}$ onto the set $\left(A_{23}\right)_{\theta^{\prime}}$ of all $\theta^{\prime}$-orbits, satisfying $s(w)=|\varepsilon(w)|$ (i.e., $\varepsilon$ preserves sizes).

Define a bijection $\delta: E \rightarrow A_{234}$ as follows: For each $w \in W^{\prime}$, set

$$
\delta\left(e_{w}\right)=a_{\varepsilon(w)},
$$

where $e_{w} \in E$ and $a_{\varepsilon(w)} \in A$ are guaranteed by (4.2'.4) and 4.2(v), respectively. Thus, $(*)$ defines $\delta \mid E_{2}$ as a bijection of $E_{2}$ onto $A_{2}$.

Extend $\delta$ to $E_{2} \cup E_{3}=\bigcup_{w \in W^{\prime}} E_{w}$ by the requirement:

$$
\theta^{\prime} \delta(e)=\delta \omega(e), \quad e \in E_{2} \cup E_{3} .
$$

By $(* *), \delta^{\prime}=\delta \mid E_{2} \cup E_{3}$ is a bijection of $E_{2} \cup E_{3}$ with $A_{23}=\bigcup_{D \in\left(A_{23}\right)_{\theta^{\prime}}} D$, and letting $\omega^{\prime}=\omega \mid E_{2} \cup E_{3}$ we have $\theta^{\prime}=\delta^{\prime} \omega^{\prime}\left(\delta^{\prime}\right)^{-1}$.

By $\left(4.2^{\prime} .1\right)$ and $\left(4.2^{\prime} .2\right)$ we have $\left|E_{4}\right|=\left|t_{W^{\prime \prime}}\right|=\left|t_{1}^{++}\right|=\left|A_{4}\right|$. Let $\delta \mid E_{4}$ be an arbitrary bijection of $E_{4}$ with $A_{4}$. Define $\xi^{\prime \prime} \in A_{234}, \eta^{\prime \prime} \in A_{34}, \zeta^{\prime \prime} \in A_{4}$ by

$$
\begin{aligned}
& \zeta^{\prime \prime}=\delta \omega^{\prime \prime} \delta^{-1}, \quad \text { where } \omega^{\prime \prime}=\omega \mid E_{4}, \\
& \eta^{\prime \prime}=\delta v^{\prime \prime} \delta^{-1}, \quad \text { where } v^{\prime \prime}=v \mid E_{3} \cup E_{4}, \\
& \xi^{\prime \prime}=\left(1_{2}+\eta^{\prime \prime}\right)\left(\theta^{\prime}+\zeta^{\prime \prime}\right) .
\end{aligned}
$$

Since $E_{2}=\left\{e_{w}: w \in W^{\prime}\right\}$ we have $v \mid E_{2}=1_{E_{2}}$ by (4.2'.3), (4.2'.4) and Proposition 2.1. Thus $\delta v \delta^{-1}=\delta\left(1_{E_{2}}+v^{\prime \prime}\right) \delta^{-1}=1_{2}+\eta^{\prime \prime}$. Similarly, $\delta \omega \delta^{-1}=$ $\delta\left(\omega^{\prime}+\omega^{\prime \prime}\right) \delta^{-1}=\theta^{\prime}+\zeta^{\prime \prime}$. Hence we have

$$
\xi^{\prime \prime}=\delta v \omega \delta^{-1}=\delta \beta \delta^{-1} .
$$

${ }^{1}$ By $\S 2$, whenever $v$ has degree $2, E_{v}$ is a singleton $\{e\}$, where $e$ is the unique edge along which one can travel from $v$ with a white region $w$ on the right-hand side. 
Thus, $\overline{\xi^{\prime \prime}}=\bar{\beta}=t_{B}=r^{++}, \overline{\eta^{\prime \prime}}=\overline{v^{\prime \prime}}=t_{V^{\prime}}=s^{++}$and $\overline{\zeta^{\prime \prime}}=\overline{\omega^{\prime \prime}}=t_{W^{\prime \prime}} t_{1}^{++}$. (4.2.1) and (4.2.2) are established and Proposition 4.2 follows.

The rest of this paper is dedicated to the Proof of Proposition 4.2' . Let $r^{++}, s^{++}, t_{0}^{++}, t_{1}^{++}$satisfy (i)-(iii) of Proposition 4.2 , and let

$$
\begin{array}{ll}
r_{0}^{++}=\sum_{3 \leq n} r(n) \cdot n^{*}, & r_{1}^{++}=r(1) \cdot 1^{*}+r(2) \cdot 2^{*}, \\
s_{0}^{++}=\sum_{3 \leq n} s(n) \cdot n^{*}, & s_{1}^{++}=s(1) \cdot 1^{*}+s(2) \cdot 2^{*},
\end{array}
$$

so that

$$
r^{++}=r_{0}^{++}+r_{1}^{++}, \quad s^{++}=s_{0}^{++}+s_{1}^{++} .
$$

By (ii) and (iii) of Proposition 4.2 there are three sequences of integers:

$$
b=\left(l_{i}\right)_{i \in \mathbb{N}}, \quad v=\left(m_{j}\right)_{j \in \mathbb{N}}, \quad w=\left(n_{k}\right)_{k \in \mathbb{N}}
$$

satisfying $3 \leq l_{i}, m_{j}(i, j \in \mathbb{N})$ and $7 \leq n_{k}(k \in \mathbb{N})$ such that

$$
r_{0}^{++}=\sum_{i \in \mathbb{N}} l_{i}^{*}, \quad s_{0}^{++}=\sum_{j \in \mathbb{N}} m_{j}^{*}, \quad t_{0}^{++}=\sum_{k \in \mathbb{N}} n_{k}^{*} .
$$

Also, there are disjoint (possibly empty) subsets $T_{B}, T_{V}, T_{W} \subseteq \mathbb{N}$ and a $T_{B}$ sequence $b^{\prime}=\left(l_{i}^{\prime}\right)_{i \in T_{B}}$, a $T_{V}$-sequence $v^{\prime}=\left(m_{j}^{\prime}\right)_{j \in T_{V}}$ and a $T_{W}$-sequence $w^{\prime}=\left(n_{k}^{\prime}\right)_{k \in T_{w}}$ of positive integers such that

$$
r_{1}^{++}=\sum_{i \in T_{B}} l_{i}^{\prime *}, \quad s_{1}^{++}=\sum_{j \in T_{V}} m_{j}^{\prime *}, \quad t_{1}^{++}=\sum_{k \in T_{W^{\prime}}} n_{k}^{\prime *}
$$

(so $l_{i}^{\prime}, m_{j}^{\prime} \in\{1,2\}$ for $i \in T_{B}, j \in T_{W}$ ).

We shall construct a bicolored planar map $M=(V, E, B, W)$ with partitions $\left\{V_{0}^{\prime}, V_{1}^{\prime}, V^{\prime \prime}\right\}$ of $V,\left\{B_{0}, B_{1}\right\}$ of $B,\left\{W^{\prime}, W^{\prime \prime}\right\}$ of $W$ and a bijection $f: W^{\prime} \rightarrow V^{\prime \prime}$ such that:

(4.2".1) $t_{B_{0}}=r_{0}^{++}, t_{B_{1}}=r_{1}^{++}, t_{V_{0}^{\prime}}=s_{0}^{++}, t_{V_{1}^{\prime}}=s_{1}^{++}, t_{W^{\prime}}=t_{0}^{++}, t_{W^{\prime \prime}}=$ $t_{1}^{++}$.

$\left(4.2^{\prime \prime} .2\right)$ If $w \in W^{\prime}$ then $f(w) \in V^{\prime \prime}$ is a vertex of degree 2 that belongs to $\operatorname{cl}(w)$.

Proposition $4.2^{\prime}$ then follows. (Define $E_{2}=\bigcup_{v \in V^{\prime \prime}} E_{v}, E_{3}=\left(\bigcup_{w \in W^{\prime}} E_{w}\right)-$ $E_{2}, E_{4}=E-\left(E_{2} \cup E_{3}\right), V^{\prime}=V_{0}^{\prime} \cup V_{1}^{\prime}$.)

Outline of the construction of $M . M$ will be obtained as the increasing union of maps $M_{n}$, where $M_{n}$ is drawn on the closed disk $D_{n}$ of radius $n$ centered at the origin. The inductive extension of $M_{n}$ to $M_{n+1}$ involves the addition of extra black regions, extra vertices and extra white regions. The sizes of black and white regions are dictated mostly by the sequences $b, w$, and the fresh vertices lie mostly on the circle $C_{n+1}$ of radius $n+1$ centered at the origin. 
In the process of extending $M_{n}$ to $M_{n+1}$, vertices on $C_{n}$ obtain their degrees as dictated by the list $v$. As a rule, a white region $w$ introduced during the extension is provided with the vertex $f(w)=v_{w}$ which lies strictly between the circles $C_{n}$ and $C_{n+1}$, and supports a black triangular region with apex $v_{w}$ with a circular arc-a segment of $C_{n+1}$-as an opposite side (see Figure 2).

For the above inductive construction to proceed smoothly, the occurrences of regions of small size and vertices of small degree should be limited. Such occurrences are forced upon us by the need to construct the black regions in $B_{1}$, the vertices in $V_{1}^{\prime}$ (which are of size 1 or 2) and possibly some of the white regions in $W^{\prime \prime}$ (which are of arbitrary finite size). We overcome this difficulty by constructing these map-components one at a time-that is, at most one at an inductive extension of $M_{n}$ to $M_{n+1}$.

The construction of a map-element from $B_{1} \cup V_{1}^{\prime} \cup W^{\prime \prime}$ is referred to in the sequel as accomplishing a task. The tasks are in one-to-one correspondence with the set $T=T_{B} \cup T_{V} \cup T_{W} \subseteq \mathbb{N}$. We take care of the first task in proceeding from $M_{1}$ to $M_{2}$, and if a task is accomplished in the transition from $M_{n}$ to $M_{n+1}$, the next one is accomplished in the transition from $M_{n+1}$ to $M_{n+2}$ or from $M_{n+2}$ to $M_{n+3}$. Thus, the $n$th task is already accomplished in $M_{2 n}$, hence all tasks are eventually accomplished in $M$.

Details. We identify for the sake of brevity the plane with the set of complex numbers $\mathbb{C}$. For $r>0$, let $U_{r}=\{z \in \mathbb{C}:|z|<r\}, C_{r}=\{z \in \mathbb{C}:|z|=r\}$ and $D_{r}=U_{r} \cup C_{r}$. For a subset $X \subseteq \mathbb{C}$ let $[X]$ denote its convex closure. For $x_{1}, \ldots, x_{n} \in \mathbb{C}$ let $\left[x_{1}, \ldots, x_{n}\right]=\left[\left\{x_{1}, \ldots, x_{n}\right\}\right]$. Thus $[x, y]$ is the line segment connecting $x$ and $y$.

Let $x, y \in C_{r}$, say $x=r e^{i \varphi}, y=r e^{i \psi}, 0 \leq \psi-\varphi<2 \pi$. Then $\lfloor x, y\rfloor=$ $\left\{r e^{i x}: \varphi \leq x \leq \psi\right\}$ denote the arc on $C_{r}$ leading from $x$ to $y$ in the positive sense, i.e., counterclockwise. $\operatorname{cl}(X)$ and $\operatorname{Int}(X)$ denote as usual the closure and the interior of $X \subseteq \mathbb{C}$.

Let $M=(V, E, B, W)$ be any bicolored planar map, and let $r$ be a positive real number. Define $V^{r}=V \cap D_{r}, E^{r}=\left\{e \cap D_{r}: e \in E\right\}, B^{r}=\left\{b \cap D_{r}: b \in B\right\}$, $W^{r}=\left\{w \cap D_{r}: w \in W\right\}$ and set $M^{r}=\left(V^{r}, E^{r}, B^{r}, W^{r}\right)$. We have $C_{r} \subseteq \bigcup E$ if and only if $C_{r} \subseteq \bigcup E^{r}$. If $C_{r} \subseteq \bigcup E^{r}$ we say that $M^{r}$ is an r-disk-map or, briefly, that $M^{r}$ is an $r$-map.

Assume that $M^{r}$ is an $r$-map. Then $\left(V^{r}, E^{r}\right)$ is obviously a planar graph, $B^{r} \subseteq B, W^{r} \subseteq W$. We call $v \in V^{r}$ an inner vertex iff $|v|<r$, and a rim vertex if $|v|=r$. Inner vertices have the same (even) degree in $M$ and in $M^{r}$, and the degree of a rim vertex is at least 2 .

The required map $M=(V, E, B, W)$ will be defined as the increasing union of disk maps, namely, its restrictions $M^{n}=\left(V^{n}, E^{n}, B^{n}, W^{n}\right)$ to the disks $D_{n}$. To help verify $\left(4.2^{\prime \prime} .1\right)$ and $\left(4.2^{\prime \prime} .2\right)$ we shall further specify partitions $\left\{{ }^{n} V_{0}^{\prime},{ }^{n} V_{1}^{\prime},{ }^{n} V^{\prime \prime}\right\}$ of $V^{n}-C_{n},\left\{{ }^{n} B_{0},{ }^{n} B_{1}\right\}$ of $B^{n}$ and $\left\{{ }^{n} W^{\prime},{ }^{n} W^{\prime \prime}\right\}$ of $W^{n}$, which eventually define the partitions $\left\{V_{0}^{\prime}, V_{1}^{\prime}, V^{\prime \prime}\right\},\left\{B_{0}, B_{1}\right\}$ and $\left\{W^{\prime}, W^{\prime \prime}\right\}$ 
of $V, B, W$ respectively. In fact, ${ }^{n} V_{1}^{\prime},{ }^{n} B_{1},{ }^{n} W^{\prime \prime}$ will be obtained as established tasks, as follows.

For $p \in T=T_{B} \cup T_{V} \cup T_{W}$, we shall define in the course of the construction the pth task $T(p)$ to be a particular map element-a black region of size $l_{p}^{\prime}$ if $p \in T_{B}$, a vertex of size $m_{p}^{\prime}$ (i.e., degree $2 m_{p}^{\prime}$ ) if $p \in T_{V}$, and a white region of size $n_{p}^{\prime}$ if $p \in T_{W}$. The task $T(p)$ will always have specified task vertex $v(p)$ associated with it. If $p \in T_{B} \cup T_{W}$ then $v(p)$ belongs to $\mathrm{cl} T(p)$, and if $p \in T_{V}$ then actually $T(p)=v(p)$.

It follows from the fact that $M^{n}$ is a disk-map, that for every $n \in \mathbb{N}, C_{n}$ is a union of edges in $E$, in fact in $E^{n}$. In addition, the set of positive integers $\mathbb{N}=\{1,2, \ldots\}$ will be a subset of $V$, and its convex closure, the ray $[1, \infty)$, will also occur as a union of edges in $E$. In general task vertices $v(p)$ will occur on this ray as half-integers, $v(p)=n_{p}+\frac{1}{2}$ for some $n_{p} \in \mathbb{N}$. (The only exceptions are some of the tasks $T(p)$ with $p \in T_{V}, m_{p}^{\prime}=1$; i.e., some vertices in $V_{1}^{\prime}$ of degree 2.)

We say that a black region $b$ (a vertex $v$; a white region $w$ ) accomplishes the integer $k$ if $s(b)=k(s(v)=k ; s(w)=k)$.

Base of the inductive construction. Let $M^{1}=\left(V^{1}, E^{1}, B^{1}, W^{1}\right)$ where

$$
\begin{gathered}
V^{1}=\left\{v_{1}=1, v_{2}, \ldots, v_{l_{1}}\right\}, \quad \text { with } v_{k}=e^{\left(2 \pi i / l_{1}\right) \cdot(k-1)}, k=1, \ldots, l_{1}, \\
E^{1}=\left\{e_{1}, \ldots, e_{l_{1}}\right\}, \quad \text { with } e_{k}=\left\lfloor v_{k}, v_{k+1}\right\rfloor, k=1, \ldots, l_{1},\left(v_{l_{1}+1}=v_{1}\right), \\
B^{1}=\left\{U_{1}\right\}, \quad W^{1}=\varnothing .
\end{gathered}
$$

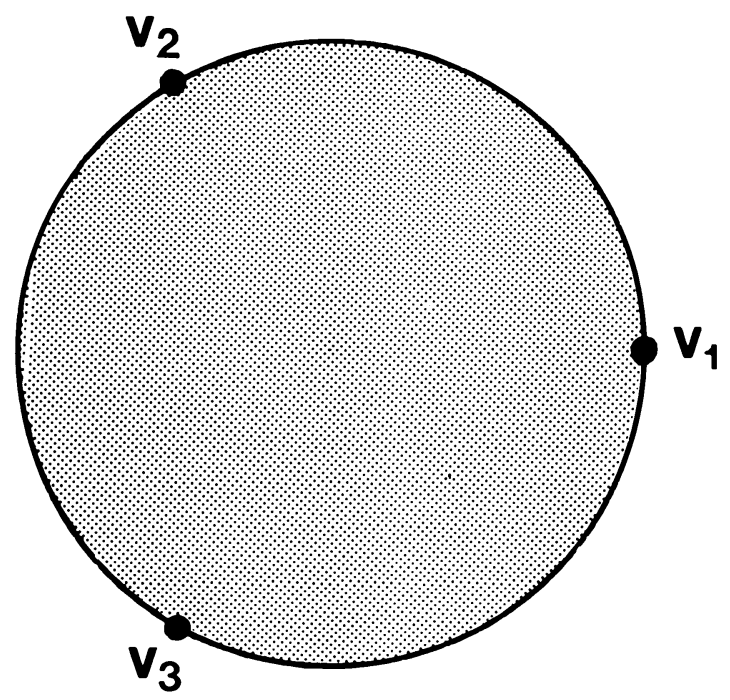

Figure 5. $M_{1}$ with $l_{1}=3$ 
We further let:

$$
{ }^{1} V_{0}^{\prime}=V^{1}, \quad{ }^{1} V_{1}^{\prime}={ }^{1} V^{\prime \prime}=\varnothing, \quad{ }^{1} B_{0}=B^{1}, \quad{ }^{1} B_{1}=\varnothing, \quad W^{\prime}=W^{\prime \prime}=\varnothing .
$$

Thus, $U_{1}$ accomplishes $l_{1}$ and let $b^{(1)}$ denote the sequence $\left(l_{i}\right)_{i \in \mathrm{N}-\{1\}}$. Let also $v^{(1)}=v, w^{(1)}=w$. Let $t^{(1)}=t=\left(t_{p}\right)_{p \in T}$ denote the task-sequence $b^{\prime} \cup v^{\prime} \cup w^{\prime}$ (i.e., $t_{p}=l_{p}^{\prime}$ if $p \in T_{B}, t_{p}=m_{p}^{\prime}$ if $p \in T_{V}, t_{p}=n_{p}^{\prime}$ if $p \in T_{W}$ ).

Induction hypothesis. Assume that $M^{n}=\left(V^{n}, E^{n}, B^{n}, W^{n}\right)$ is already defined, and so are the sequences of positive integers $b^{(n)}, v^{(n)}, w^{(n)}, t^{(n)}$. These are obtained from the sequences $b, v, w, t$ by omitting a finite number of terms from each, namely, the terms accomplished in $M_{n}$; that is, the terms omitted from the sequence $b \quad(v ; w ; t)$ stand in one-to-one correspondence with the regions in ${ }^{n} B_{0}$ (the set ${ }^{n} V_{0}^{\prime}$ of inner vertices of $V_{n}$; the set ${ }^{n} W^{\prime}$ of white region of $M_{n}$; the task-set $\left.{ }^{n} B_{1} \cup{ }^{n} V_{1}^{\prime} \cup{ }^{n} W^{\prime \prime}\right)$ that accomplish them. Also, each $w \in W^{\prime}$ has an inner vertex $v_{w} \in{ }^{n} V^{\prime \prime}$ of degree two in its closure, and ${ }^{n} V^{\prime \prime}=\left\{v_{w}: w \in W^{\prime}\right\}$.

Let $v_{1}=n, v_{2}, \ldots, v_{q} \in V^{n}$ enumerate the rim vertices of $M_{n}$ in the positive sense, and let $2 \leq d_{n}\left(v_{i}\right)$ denote the degree of $v_{i}$ in $M_{n}$. We assume that one of the following two cases hold:

Case a. $d_{n}\left(v_{i}\right) \leq 4$ for $i=1, \ldots, q$.

Case b. $d_{n}\left(v_{1}\right)=5$ and $d_{n}\left(v_{i}\right) \leq 4$ for $i=2, \ldots, q$.

Induction Step. The extension of $M^{n}$ to $M^{n+1}$ is made in several steps. In general, the 1-skeleton of the extension (i.e., the union of its edges) is defined, being divided by inserted vertices into edges later on, to obtain the required sizes for fresh map elements. The actual extension depends on whether $M_{n}$ obeys Case $a$ or Case $b$ and whether any tasks are left. Thus, we distinguish two cases:

Case 1. Case b occurs, or no tasks are left.

Case 2. Case a occurs, and some tasks are left.

In Case 1 a "smooth" extension leads to $M_{n+1}$, where Case a occurs. In Case 2 , the first nonaccomplished task is accomplished in the course of the extension.

We turn to the details. Recall that $v_{1}=n, v_{2}, \ldots, v_{q} \in C_{n}$ are the rim vertices of $M^{n}$, and $d_{n}\left(v_{i}\right)$ is the degree of $v_{i}$ in $M^{n}, i=1, \ldots, q$. We let $v_{q+1}=v_{1}$ and $e_{i}=\left\lfloor v_{i}, v_{i+1}\right\rfloor, i=1, \ldots, q, e_{q+1}=e_{1}$. For $i=1, \ldots, q$ we define $X_{i} \in\{\underline{B}, \underline{W}\}$ as follows: $X_{i}=\underline{B}\left(X_{i}=\underline{W}\right)$ iff $e_{i}$ lies in the closure of a white (black) region of $M_{n}$. Thus, $X_{i}=\underline{B}\left(X_{i}=\underline{W}\right)$ iff the region of $M$ touching $e_{i}$ and lying outside $D_{n}$ should be black (white). We now proceed by the two cases as follows:

Case 1. $d_{n}\left(v_{1}\right)=5, d_{n}\left(v_{i}\right) \leq 4$ for $i=1,2, \ldots, q$ or $t^{(n)}$ is an empty sequence. 
Step 1.1 (assigning sizes to new map elements supporting edges on $C_{n}$ ). Declare $s\left(v_{1}\right), \ldots, s\left(v_{q}\right)$ to be the first $q$ members of the sequence $v^{(n)}$ (so $\left.s\left(v_{i}\right) \geq 3, i=1, \ldots, q\right)$. Let $f_{i}$ denote the $M^{n+1}$ region lying outside $D_{n}$ and having $e_{i}$ in its closure (to be determined in full later), $i=1, \ldots, q$. Let $g_{1}, \ldots, g_{q^{\prime}}\left(h_{1}, \ldots, h_{q^{\prime \prime}}\right)$ be the subsequence of $f_{1}, \ldots, f_{q}$ consisting of all $f_{i}$ with $X_{i}=\underline{B}\left(X_{i}=\underline{W}\right)$. Let $s\left(g_{1}\right), \ldots, s\left(g_{q^{\prime}}\right)$ be the first $q^{\prime}$ members of the sequence $b^{(n)}$, and let $s\left(h_{1}\right), \ldots, s\left(h_{q^{\prime \prime}}\right)$ be the first $q^{\prime \prime}$ members of the sequence $w^{(n)}$ (so $s\left(g_{i}\right) \geq 3, i=1, \ldots, q^{\prime}$, and $\left.s\left(h_{i}\right) \geq 6,{ }^{2} i=1, \ldots, q^{\prime \prime}\right)$.

Step 1.2 (determination of all edges connecting $C_{n}$ with $C_{n+1}$ ). Declare $C_{n+1}$ to be part of the 1-skeleton of $M_{n+1}$. Declare $u_{i}=\left(1+\frac{1}{n}\right) v_{i} \in C_{n+1}$ to be a vertex in $V^{n+1}$, and $\left[v_{i}, u_{i}\right]$ to be an edge in $E^{n+1}$. Let $1 \leq i \leq q$, and consider the number $d_{i}^{\prime}=2 s\left(v_{i}\right)-d_{n}\left(v_{i}\right)$ (which may be called "the degree of $v_{i}$ outside $D_{n}$ "). Since $s\left(v_{i}\right) \geq 3, d_{n}\left(v_{i}\right) \leq 4$ for $i=2, \ldots, q$, we have $d_{i}^{\prime} \geq 2$ for $i=2, \ldots, q$. In general, $d^{\prime}\left(v_{1}\right) \geq 2$ as well. If, however, Case $\mathrm{b}$ holds, then $d_{n}\left(v_{1}\right)=5$, so if in addition $s\left(v_{1}\right)=3$, we have $d^{\prime}\left(v_{1}\right)=1$. We proceed according to two possible options.

Case 1.2.1. $d_{1}^{\prime} \geq 2$, or $s\left(f_{1}\right)>3$.

(1.2.1.1) For $i=1, \ldots, q$ choose a vertex $u_{i}^{+} \in\left\lfloor u_{i}, u_{i+1}\right\rfloor$ (where $u_{q+1}=u_{1}$ ) and an edge $e_{i}^{\prime} \in E^{n+1}$ connecting $v_{i}$ to $u_{i}^{+}$as follows: If $s\left(f_{i}\right)>3$, let $u_{i}^{+} \neq$ $u_{i}, u_{i+1}$ be an internal point of the arc $\left\lfloor u_{i}, u_{i+1}\right\rfloor$ such that $\left[v_{i}, u_{i}^{+}\right] \cap U_{n}=\varnothing$.

Let $e_{i}^{\prime}=\left[v_{i}, u_{i}^{+}\right]$. If $s\left(f_{i}\right)=3$ (in which case $X_{i}=\underline{B}$, i.e., $f_{i}$ is to be a black region) let $u_{i}^{+}=u_{i+1}$, and let $e_{i}^{\prime}$ be a straight line segment or any circular arc connecting $v_{i}$ to $u_{i}^{+}$meeting $D_{n}$ at $v_{i}$ and lying in $D_{n+1} \cap\left[\left\{v_{i}, v_{i+1}\right\} \cup\right.$ $\left.\left\lfloor u_{i}, u_{i+1}\right\rfloor\right]$, that meets $\left[v_{i}, u_{i}\right]$ at nonzero angle.

(1.2.1.2) Declare $d_{i}^{\prime}-2$ distinct points other than $u_{i}, u_{i}^{+}$on the arc $\left\lfloor u_{i}, u_{i}^{+}\right\rfloor$ to be vertices in $V^{n+1}$, and the line segments connecting them to $v_{i}$ to be edges in $E^{n+1}$ (these new points $u \in\left\lfloor u_{i}, u_{i}^{+}\right\rfloor$are chosen so that the line segment $\left[v_{i}, u\right]$ does not intersect $\left.e_{i}^{\prime}\right)$.

(1.2.1.3) Let $\tilde{f}_{i}$ denote the open region bounded by the curves $e_{i}^{\prime}, e_{i}=$ $\left\lfloor v_{i}, v_{i+1}\right\rfloor,\left[v_{i+1}, u_{i+1}\right]$ and $\left\lfloor u_{i}^{+}, u_{i+1}\right\rfloor$ (if $\left.u_{i}^{+} \neq u_{i+1}\right)$.

Case 1.2.2. $d_{1}^{\prime}=1$ and $s\left(f_{1}\right)=3$.

(1.2.2.1) For $i=2, \ldots, q+1$ choose a vertex $u_{i}^{-} \in\left\lfloor u_{i-1}, u_{i}\right\rfloor$ (where $\left.u_{q+1}=u_{1}\right)$ and an edge $e_{i}^{\prime}$ connecting $v_{i}$ to $u_{i}^{-}$as follows: If $s\left(f_{i-1}\right)>3$, let $u_{i}^{-} \neq u_{i-1}, u_{i}$ be an internal point of $\left\lfloor u_{i-1}, u_{i}\right\rfloor$ such that $\left[v_{i}, u_{i}^{-}\right] \cap U_{n}=\varnothing$. Let $e_{i}^{\prime}=\left[v_{i}, u_{i}^{-}\right]$. If $s\left(f_{i-1}\right)=3$ (in which case $X_{i}=\underline{B}$ ) let $u_{i}^{-}=u_{i-1}$ and let $e_{i}^{\prime}$ be a straight line segment or any circular arc connecting $v_{i}$ to $u_{i}^{-}$meeting $D_{n}$ at $v_{i}$ and lying in $D_{n+1} \cap\left[\left\{v_{i}, v_{i-1}\right\} \cup\left[u_{i-1}, u_{i}\right\rfloor\right]$, that meet $\left[v_{i}, u_{i}\right]$ at nonzero angle.

${ }^{2}$ Actually, $s\left(h_{i}\right) \geq 7$; but in Case $1 s\left(h_{i}\right) \geq 6$ is sufficient. 


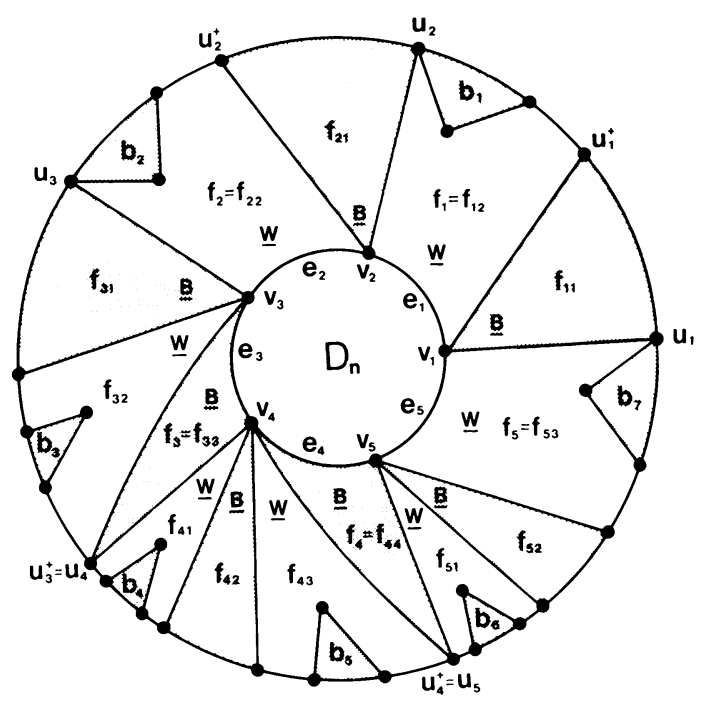

FIgURE 6. Step 1, Case 1.2.1, with $q=5, X_{1}=X_{2}=$ $X_{5}=\underline{W}, X_{3}=X_{4}=\underline{B}, d_{1}^{\prime}=d_{2}^{\prime}=2, d_{3}^{\prime}=d_{5}^{\prime}=3$, $d_{4}^{\prime}=4$.

(1.2.2.2) Declare $d_{i}^{\prime}-2$ distinct points of the arc $\left\lfloor u_{i}^{-}, u_{i}\right\rfloor$ other than $u_{i}^{-}, u_{i}$ to be vertices in $V^{n}$ and the line segments connecting them to be edges in $E^{n+1}$. (The new points $u \in\left\lfloor u_{i}^{-}, u_{i}\right\rfloor$ are chosen so that $\left[v_{i}, u\right] \cap e_{i}^{\prime}=\varnothing$.)

$(1.2 .2 .3)$ Let $\tilde{f}_{i}$ denote the open region bounded by the curves $e_{i+1}^{\prime}, e_{i}=$ $\left\lfloor v_{i}, v_{i+1}\right\rfloor,\left[v_{i}, u_{i+1}^{-}\right]$and $\left\lfloor u_{i}, u_{i+1}^{-}\right]$(if $\left.u_{i} \neq u_{i+1}^{-}\right)$.

Case 1.2.3. We now establish notation and colour for the regions obtained so far in $D_{n+1}-D_{n}$. For $i=1, \ldots, q$ and $j=1, \ldots, d_{i}^{\prime}$ we define the region $\tilde{f}_{i j}$ and colour $X_{i j} \in\{\underline{B}, \underline{W}\}$ as follows. For $X \in\{\underline{B}, \underline{W}\}$ define $X^{\prime}$ by $\underline{B}^{\prime}=\underline{W}$, $\underline{W^{\prime}}=\underline{B}$.

In Case 1.2.1, let $\tilde{f}_{i d_{i}^{\prime}}=\tilde{f}_{i}, X_{i d_{i}^{\prime}}=X_{i}$. Let $\tilde{f}_{i j-1}$ be the neighboring region to $\tilde{f}_{i j}$ to the right. Let $X_{i j-1}=X_{i j}^{\prime}$ (see Figure 6).

In Case 1.2.2, let $\tilde{f}_{i 1}=\tilde{f}_{i}, X_{i 1}=X_{i}$. Let $\tilde{f}_{i j+1}$ be the region to the left of $\tilde{f}_{i j}, X_{i j+1}=X_{i j}^{\prime}$ (see Figure 7).

Remarks. 1.2.4. The parity of the degrees $d\left(v_{i}\right)=2 s\left(v_{i}\right)$ and the fact that $M_{n}$ is properly colored provide us with the fact that

$$
X_{11}, \ldots, X_{1 d_{1}^{\prime}}, \ldots, X_{21}, \ldots, X_{2 d_{2}}, \ldots, X_{q 1}, \ldots, X_{q d_{q}^{\prime}}
$$

is an alternating sequence of $\underline{B}$ and $\underline{W}$.

1.2.5. All vertices on $C_{n+1}$ defined so far have degree $\leq 4$. The only vertices of degree 4 are some of the vertices $u_{1}, \ldots, u_{q} . u_{i}$ has degree 4 only in the following two cases: Case 1.2.1 and $s\left(f_{i-1}\right)=3$, or Case 1.2.2 and $s\left(f_{i}\right)=3$. Note that in both cases $X_{i}=\underline{B}$. 


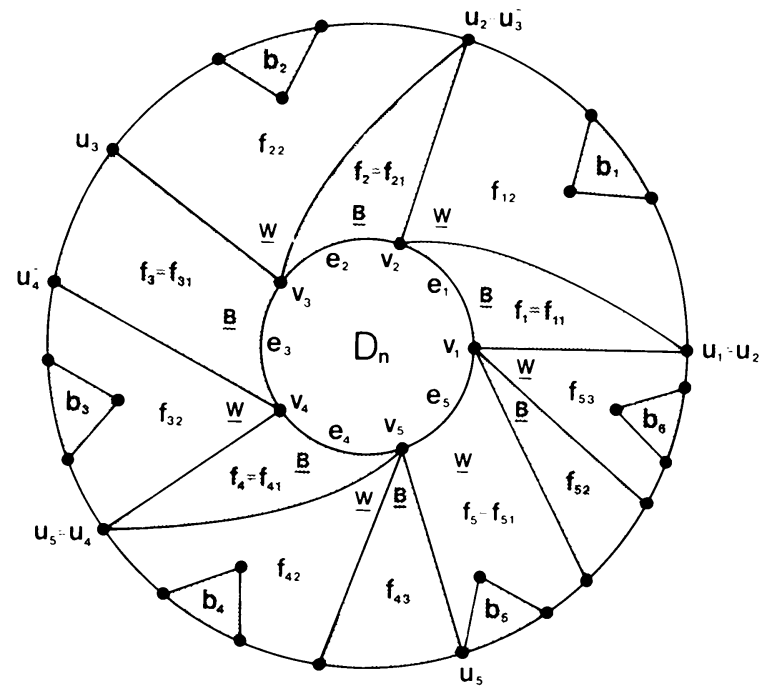

Figure 7. Step 1, Case 1.2.2, with $q=5, X_{1}=X_{2}=$ $X_{3}=X_{4}=\underline{B}, X_{5}=\underline{W}, d_{1}^{\prime}=d_{5}^{\prime}=3, d_{2}^{\prime}=d_{3}^{\prime}=d_{4}^{\prime}=$ 2 .

Step 1.3 (determination of all $M_{n+1}$-regions in $D_{n+1}-D_{n}$ ). Consider the sequence

$$
\tilde{f}_{11}, \ldots, \tilde{f}_{1 d_{1}^{\prime}}, \tilde{f}_{21}, \ldots, \tilde{f}_{2 d_{2}^{\prime}}, \ldots, \tilde{f}_{q 1}, \ldots, \tilde{f}_{q d_{q}^{\prime}}
$$

that enumerates all the regions obtained so far in the ring $D_{n+1}-D_{n}$, in a cyclic order, from the edge $[n, n+1]$ in a positive sense.

Let $\tilde{h}_{1}, \ldots, \tilde{h}_{r}$ denote the subsequence of those $\tilde{f}_{i j}$ for which $X_{i j}=\underline{W}$. We say that $\tilde{h}_{k}$ is triangular if $\operatorname{cl}\left(\tilde{h}_{k}\right) \cap C_{n}$ is a point, i.e., if $\tilde{h}_{k} \neq f_{i}$ for $i=1, \ldots, q$. Notice that $\operatorname{cl}\left(\tilde{h}_{k}\right) \cap C_{n+1}$ is a nondegenerate arc on $C_{n+1}$ (see 1.2.5), and denote it $\left\lfloor x_{k}, y_{k}\right\rfloor$. Next we choose $x_{k}^{+}, y_{k}^{-} \in\left\lfloor x_{k}, y_{k}\right\rfloor$ and $z_{k} \in \tilde{h}_{k}$ $\left(n<\left|z_{k}\right|<n+1\right)$ so that $x_{k}, x_{k}^{+}, y_{k}^{-}, y_{k}$ appear on $C_{n+1}$ in this order, $x_{k}^{+} \neq y_{k}^{-}$ and $b_{k}=\left[\left\{z_{k}\right\} \cup\left[x_{k}^{+}, y_{k}^{-}\right\rfloor\right] \subseteq \tilde{h}_{k}$. If $\tilde{h}_{k}$ is triangular, we further assume $x_{k} \neq x_{k}^{+}$and $y_{k} \neq y_{k}^{-}$. If $\tilde{h}_{k}$ is not triangular, we let $x_{k}=x_{k}^{+}, y_{k}^{-} \neq y_{k}$ if Case 1.2.1 holds and $x_{k} \neq x_{k}^{+}, y_{k}^{-}=y_{k}$ if Case 1.2.2 holds. ${ }^{3}$

We declare $x_{k}^{-}, y_{k}^{+}, z_{k}$ to be vertices in $V^{n+1},\left[z_{k}, x_{k}^{+}\right],\left[z_{k}, y_{k}^{-}\right]$to be edges in $E_{n+1}$ and $b_{k}$ to be a black region in $B^{n+1}$.

We set ${ }^{n+1} V^{\prime \prime}=\left\{z_{k}: k=1, \ldots, r\right\}$. Let $1 \leq i \leq q, 1 \leq j \leq d_{i}^{\prime}$. If $X_{i j}=\underline{B}$, we let $f_{i j}=\tilde{f}_{i j} \in B^{n+1}$. If $X_{i j}=\underline{W}$, let $\tilde{f}_{i j}=\tilde{h}_{k}$ and let $f_{i j}=\tilde{h}_{k}-\operatorname{cl}\left(b_{k}\right)$.

${ }^{3}$ This ensures that $\tilde{h}_{k}-\mathrm{cl}\left(b_{k}\right)$ has no more than six vertices defined so far in its closure, and no vertex on $C_{n+1}$ has degree exceeding 4 . 
Let $f_{i j} \in W^{n+1}$. Thus we have

$$
\begin{gathered}
B^{n+1}-B^{n}=\left\{b_{k}: k=1, \ldots, r\right\} \cup\left\{f_{i j}: X_{i j}=\underline{B}\right\}, \\
W^{n+1}-W^{n}=\left\{f_{i j}: X_{i j}=\underline{W}\right\} .
\end{gathered}
$$

We declare $B^{n+1}-B^{n} \subseteq B_{0}^{n+1}, W^{n+1}-W^{n} \subseteq W_{0}^{n+1}$.

Step 1.4 (completion of $M^{n+1}$-additional vertices on $C_{n+1}$ ).

1.4.1. Assign a size $s(b)(s(w))$ to each region of $B^{n+1}-B^{n}\left(W^{n+1}-W^{n}\right)$ other than $f_{1}, \ldots, f_{q}$, whose sizes were already determined in Step 1.1. For this purpose use the first members of the sequence $b^{(n)}\left(w^{(n)}\right)$ not assigned to map elements in 1.1. Thus, $s(b) \geq 3$ for all $b \in B^{n+1}-B^{n}$ and $s(w) \geq 7$ for all $w \in W^{n+1}-W^{n}$.

1.4.2. Add vertices on $C_{n+1}$ so that we have $s(b)=\left|E_{b}\right|$ and $s(w)=\left|E_{w}\right|$ for each $b \in B^{n+1}-B^{n}$ and $w \in W^{n+1}-W^{n}$. Declare the arcs into which $C_{n+1}$ is divided edges in $E_{n+1}$. Let

$$
\begin{gathered}
{ }^{n+1} V^{\prime \prime}-V^{n}=\left\{z_{k}: k=1, \ldots, r\right\} \subseteq U^{n+1}-D_{n}, \\
{ }^{n+1} V_{0}^{\prime}-V^{n}=V^{n+1} \cap C_{n+1}, \quad{ }^{n+1} V_{1}^{\prime}=\varnothing .
\end{gathered}
$$

The induction step is complete. Notice that every vertex on $C_{n+1}$ has degree 2,3 or 4 , i.e., Case a occurs.

Case 2. $d_{n}\left(v_{i}\right) \leq 4, i=1, \ldots, q$, and $t^{(n)}$ is not an empty sequence. Let $t_{p}$ be the first member of $t^{(n)}$. The $p$ th task $T(p)$ will be accomplished in $D_{n+1}-D_{n}$, in the course of this inductive step. We shall denote the possible tasks to be considered as $T(p)$ as follows:

Task B.k.: $p \in T_{B}$ and $t_{p}=k \quad(k \in\{1,2\})$,

Task V.k.: $p \in T_{V}$ and $t_{p}=k \quad(k \in\{1,2\})$,

Task W.k.: $p \in T_{W}$ and $t_{p}=k \quad(k \in \mathbb{N})$.

We proceed in steps:

Step 2.1 (assigning sizes to map elements supporting edges in $C_{n+1}$ ). Same as Step 1.1. Notice that $d_{i}^{\prime} \geq 2, i=1, \ldots, q$.

Step 2.2 (division of the ring $D_{n+1}-D_{n}$ into compartments). Declare $C_{n+1}$ to be part of the 1-skeleton of $M_{n+1}$. Declare $u_{i}=\left(1+1 / n_{i}\right) v_{i} \in C_{n+1}$ to be a vertex in $V^{n+1}$, and $\left[v_{i}, u_{i}\right]$ to be edges in $E^{n+1}, i=2, \ldots, q$. Declare $\left[v_{1}, u_{1}\right]$ to be part of the 1-skeleton of $M_{n+1}$.

Let us call the region enclosed by the curves $\left\lfloor v_{q}, v_{2}\right\rfloor,\left[v_{2}, u_{2}\right],\left\lfloor u_{q}, u_{2}\right\rfloor$, $\left[v_{q}, u_{q}\right]$ the task-region.

Step 2.3 (accomplishment of the task). This step splits into cases dictated by the following parameters:

(i) The task to be accomplished. 


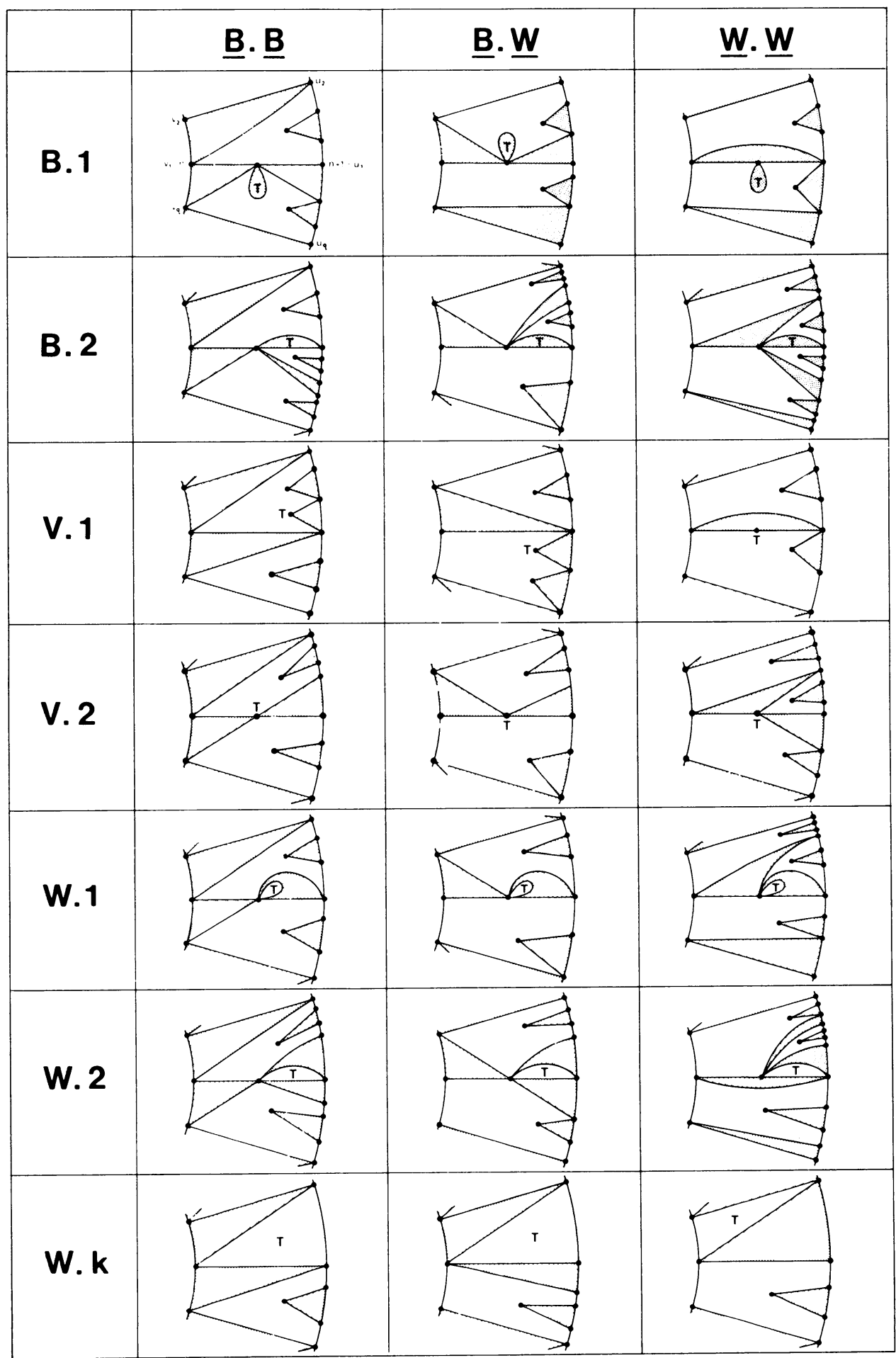

FIGURE 8 
(ii) The values $X_{1}, X_{q} \in\{\underline{B}, \underline{W}\}$.

(ii) Sizes preassigned already to map elements in the task region, mainly $d_{1}^{\prime}=2 s\left(v_{1}\right)-d_{n}\left(v_{1}\right), s\left(f_{1}\right), s\left(f_{q}\right)$.

We shall describe this step by displaying the actual solution for each of the possible tasks, under the most tight assumption about (iii), namely: The size of a nontask black region is 3 . The size of a nontask vertex is 3 (i.e., its degree is 6 ) and the size of a nontask white region is at most 7 (nontask white regions always possess an arc (possibly degenerated to a point) on $C_{n+1}$ as part of their boundary, so their size is always augmentable by adding vertices on that arc). These cases are displayed in Figure 8. The other (easier) cases can be safely left to the reader.

With the mentioned assumptions about sizes, we denote the cases as follows: Let $X, Y \in\{\underline{B}, \underline{W}\}$. B.k.X.Y (V.k.X.Y, W.k.X.Y) will stand for task B.k (V.k, $W . k)$ with $X_{1}=X, X_{q}=Y$. In addition, the assumption $d_{1}^{\prime}=1$ or $d_{1}^{\prime}=2$ is utilized for the demonstration. In fact, $d_{1}^{\prime}=1$ is ruled out, as we are in Case a; but obviously, $d_{1}^{\prime}>1$ only facilitates the construction. In each figure, the letter " $T$ " denotes the task. We note that once we display a figure for U.k. $B, W$ $(U=B, V$ or $W$ ), a figure for $U \cdot k . W \cdot B$ will be obtained by reflecting on the real axis.

Step 2.4 (completion of $M_{n+1}$ ). Proceed to define $M_{n+1}$ outside the task region as in Case 1, by suitably modifying Steps 1.2-1.4.

\section{BIBLIOGRAPHY}

[ACM] Z. Arad, D. Chillag and G. Moran, Groups with a small covering number, Chapter 4 of $[\mathrm{AH}]$.

[AH] Z. Arad and M. Herzog, Products of conjugacy classes in groups, Lecture Notes in Math., vol. 1112, Springer, 1985.

[B] E. Bertram, On a theorem of Schreier and Ulam for permutations, J. Algebra 24 (1973), 316322.

[Bo] G. Boccara, Cycles comme produit de deux permutations de classes donnees, Discrete Math. 38 (1982), 129-142.

[D1] M. Droste, Products of conjugacy classes of the infinite symmetric groups, Discrete Math. 47 (1983), 35-48.

[D2] _ Cubes of conjugacy classes covering the infinite symmetric group, Trans. Amer. Math. Soc. 288 (1985), 381-393.

[D3] __ Squares of conjugacy classes in the infinite symmetric group, Trans. Amer. Math. Soc. 303 (1987), 503-515.

[DG1] M. Droste and R. Gobel, On a theorem of Baer, Schreier and Ulam for permutations, J. Algebra 58 (1979), 282-290.

[DG2] __ Products of conjugate permutations, Pacific J. Math. 92 (1981), 47-60.

[Dv] Y. Dvir, Covering properties of permutation groups, Chapter 4 of [AH].

[G] A. B. Gray, Infinite symmetric and monomial groups, Ph.D. Thesis, New Mexico State University, Las Cruces, New Mexico, 1960.

[H] P. Hall, Some constructions for locally finite groups, J. London Math. Soc. 34 (1959), 305-319.

[M1] G. Moran, The algebra of reflections of an infinite set, Notices Amer. Math. Soc. 73T (1973), A193. 
[M2] $\ldots$, The product of two reflection classes of the symmetric group, Discrete Math. 15 (1976), 63-77.

[M3] _ Parity features for classes of the infinite symmetric group, J. Combin. Theory Ser A 33 (1982), 82-98.

[M4] _ Of planar Eulerian graphs and permutations, Trans. Amer. Math. Soc. 287 (1985), 323341.

[M5] _ The product of conjugacy classes in some infinite simple groups, Israel J. Math. 50 (1985), 54-74.

[M6] _ Products of involution classes in infinite symmetric groups, Trans. Amer. Math. Soc. 307 (1988), 745-762.

[O] O. Ore, Some remarks on commutators, Proc. Amer. Math. Soc. 2 (1951), 307-314.

[S] W. R. Scott, Group theory, Prentice-Hall, Englewood Cliffs, N.J., 1964.

Department of Mathematics, University of Haifa, Haifa 31999, IsRael 